\title{
OPTIMIZATION FOR DESIGN AND OPERATION OF NATURAL GAS TRANSMISSION NETWORKS
}

\author{
A Thesis \\ by \\ SEBNEM DILAVEROGLU
}

\author{
Submitted to the Office of Graduate Studies of \\ Texas A\&M University \\ in partial fulfillment of the requirements for the degree of \\ MASTER OF SCIENCE
}

\begin{abstract}
Approved by:
Chair of Committee, Halit Üster

Committee Members, Sıla Çetinkaya

H. Neil Geismar

Department Head, César O. Malavé
\end{abstract}

December 2012

Major Subject: Industrial Engineering

Copyright 2012 Sebnem Dilaveroglu 


\begin{abstract}
This study addresses the problem of designing a new natural gas transmission network or expanding an existing network while minimizing the total investment and operating costs. A substantial reduction in costs can be obtained by effectively designing and operating the network. A well-designed network helps natural gas companies minimize the costs while increasing the customer service level. The aim of the study is to determine the optimum installation scheduling and locations of new pipelines and compressor stations. On an existing network, the model also optimizes the total flow through pipelines that satisfy demand to determine the best purchase amount of gas.

A mixed integer nonlinear programming model for steady-state natural gas transmission problem on tree-structured network is introduced. The problem is a multiperiod model, so changes in the network over a planning horizon can be observed and decisions can be made accordingly in advance. The problem is modeled and solved with easily accessible modeling and solving tools in order to help decision makers to make appropriate decisions in a short time. Various test instances are generated, including problems with different sizes, period lengths and cost parameters, to evaluate the performance and reliability of the model. Test results revealed that the proposed model helps to determine the optimum number of periods in a planning horizon and the crucial cost parameters that affect the network structure the most.
\end{abstract}




\section{DEDICATION}

To my family 


\section{ACKNOWLEDGEMENTS}

I would like to express my sincere gratitude to all those people who somehow contributed to this research. First and foremost, I would like to thank my advisor,

Dr. Halit Üster for his guidance and for his encouragement. I thank him for teaching me and for supporting me.

Thanks are also extended to the other committee members, Dr. Sıla Çetinkaya and Dr. Neil Geismar. Their suggestions, comments and support were helpful and precious.

I am deeply grateful to Yavuz Yılmaz and Gürcan Öz, system operation engineers at Petroleum Pipeline Corporation, Turkey, who taught me everything about natural gas pipeline systems. They were always open for discussions and answered all my questions with patience.

Most importantly, I would like to thank my family and my friends who encouraged and supported me in pursuing my dream. Without their faith and their love, I could not have come this far.

I owe special thanks to Zeynep Adıgüzel for her friendship and support through all these years. She has always been there for me.

Finally, I acknowledge BOTAS-Petroleum Pipeline Corporation for their financial support for this thesis. 


\section{TABLE OF CONTENTS}

Page

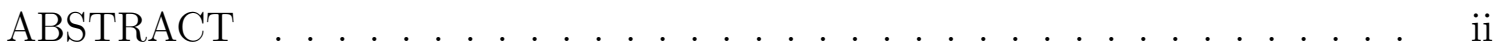

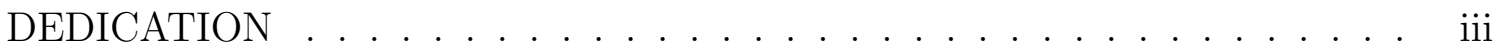

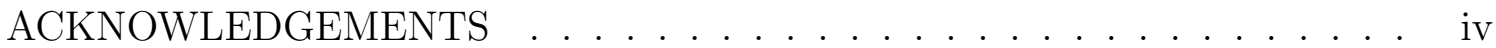

TABLE OF CONTENTS . . . . . . . . . . . . . . . . v v

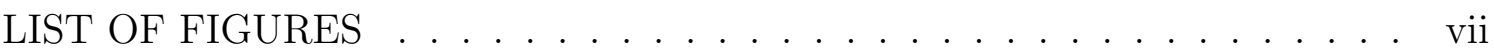

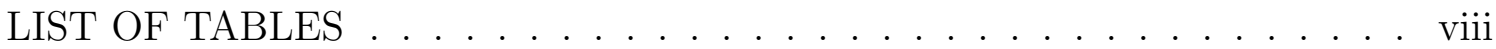

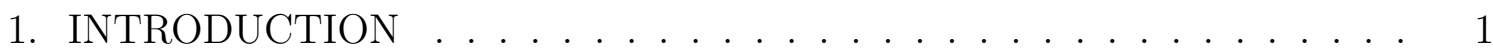

1.1 Research Objectives and Scope ... . . . . . . . . . . . 3

2. LITERATURE REVIEW . . . . . . . . . . . . . . . . . 5

3. PROBLEM DEFINITION AND MATHEMATICAL FORMULATION . . 11

3.1 Characteristics of the System . . . . . . . . . . . . . . 11

3.2 System Components . . . . . . . . . . . . . . . . . . . . . . 14

3.2.1 Pipelines.......................... 14

3.2 .2 Compressor Stations . . . . . . . . . . . . . 17

3.2 .3 Cost Structure . . . . . . . . . . . . . . . . . . 19

3.3 Modeling Assumptions . . . . . . . . . . . . . . . . 25

3.4 Network Structure . . . . . . . . . . . . . . . . . . . . . . 26

3.5 The Mathematical Model . . . . . . . . . . . . . . . . . . . 27

4. THE SOLUTION METHOD AND COMPUTATIONAL STUDY . . . . . 32

4.1 The Solution Method . . . . . . . . . . . . . . . . . 32

4.1.1 Mixed Integer Nonlinear Programming . . . . . . . . . . . . . 33

4.1.2 The Branch-and-Bound Algorithm . . . . . . . . . . . . . . 34 
4.1.3 Overview of AMPL and Bonmin . . . . . . . . . . . . . 35

4.2 Computational Study and Analysis . . . . . . . . . . . . . . 36

4.2.1 Experiment 1: Various Problem Sizes . . . . . . . . . . . . . . 40

4.2.2 Experiment 2: The Effect of the Period Lengths . . . . . . . . 48

4.2.3 Experiment 3: The Effect of Changes in the Cost Parameters . 63

5. CONCLUSIONS AND FUTURE RESEARCH DIRECTIONS . . . . . . 70

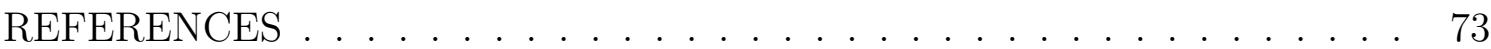




\section{LIST OF FIGURES}

Page

1.1 Natural Gas Consumption by Sector . . . . . . . . . . . . . . . . 1

3.1 Graphical Notation for the Networks . . . . . . . . . . . . . . . . 12

3.2 A Natural Gas Transmission Pipeline Network . . . . . . . . . . . . . 13

3.3 Steady Flow in Gas Pipeline . . . . . . . . . . . . . . . . . 15

3.4 Representation of Nodes and Arcs . . . . . . . . . . . . . . . . 18

3.5 Representation of Compressor Station . . . . . . . . . . . . . 18

4.1 A Network with 31 Nodes . . . . . . . . . . . . . . . . . . . 41

4.2 Solution to the 31-Node Network for 1-period . . . . . . . . . . . . . 41

4.3 Flow Rates in the 31-Node Network for 1-period . . . . . . . . . . . . 43

4.4 A Network with 66 Nodes . . . . . . . . . . . . . . . 44

4.5 Solution to the 66 -Node Network for 1-period . . . . . . . . . . 45

4.6 A Network with 97 Nodes . . . . . . . . . . . . . . 46

4.7 Solution to the $97-$ Node Network for 1-period . . . . . . . . . . . 47

4.8 Problems with Different Period Lengths . . . . . . . . . . . . . . . 49

4.9 Solution to the 1-Period Planning . . . . . . . . . . . . . 51

4.10 Solution to the 2-Period Planning . . . . . . . . . . . . 52

4.11 Solution to the 3-Period Planning . . . . . . . . . . . . . 53

4.12 Solution to the 4-Period Planning (1) . . . . . . . . . . 54

4.13 Solution to the 4-Period Planning (2) . . . . . . . . . . . 55

4.14 Solution to the 6-Period Planning . . . . . . . . . . . 56

4.15 Solution to the 12 -Period Planning . . . . . . . . . . . . 57

4.16 Annual Costs of Different Period Lengths . . . . . . . . . . . . 61

4.17 Network Structure for Problems $T_{5}$ and $T_{6} \ldots \ldots$. . . . . . . 67

4.18 Network Structure for Problems $T_{7}$ and $T_{8} \ldots \ldots$. . . . . . . 68 


\section{LIST OF TABLES}

Page

2.1 Classification of Natural Gas Network Planning Literature . . . . . 10

2.2 Characteristics of Natural Gas Optimization Problems . . . . . . . 10

3.1 Data for Cost Computation _ . . . . . . . . . . . . 20

3.2 Investment and Operating Costs $\left(10^{3} \$\right) \ldots \ldots \ldots \ldots \ldots$

3.3 Purchase Costs $\left(10^{3} \$\right) \ldots \ldots \ldots \ldots \ldots$

3.4 Transportation Costs $\left(10^{3} \$\right) \ldots \ldots \ldots \ldots \ldots \ldots$

4.1 Monthly Demand Data $(\mathrm{mmscm}) \ldots \ldots \ldots$

4.2 Pipeline Arc Lengths and Resistances . . . . . . . . . . . . . . . . . 39

4.3 Pressure Levels at Nodes in the 31-Node Network for 1-period . . . . 42

4.4 Optimal Costs and Solution Times for Different Sized Networks . . . 48

4.5 Optimal Costs and Solution Times for Different Period Lengths . . . 58

4.6 Cost Data $\left(10^{3} \$\right)(1) \ldots \ldots \ldots \ldots \ldots \ldots \ldots$

4.7 Cost Data $\left(10^{3} \$\right)(2) \ldots \ldots \ldots \ldots \ldots \ldots$

4.8 Optimal Costs and Solution Times for Different Combinations of Cost

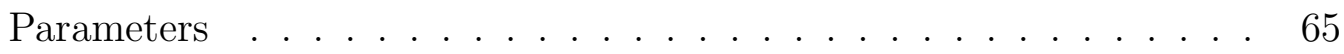




\section{INTRODUCTION}

The continual increase in the oil prices and the environmental concerns about high level of air pollution has led natural gas (NG) to become one of the important energy sources in the world. With a growing population and economy, the demand for NG has increased because of expanding industrial and commercial sectors, and households with growing income. As shown in Figure 1.1, NG is used mostly for industrial purposes and electric power production. The US Energy Information Administration reports that global NG consumption doubled from 1980 to 2010 [30] and it is expected to increase to approximately 4 trillion cubic meters in 2030 [29].
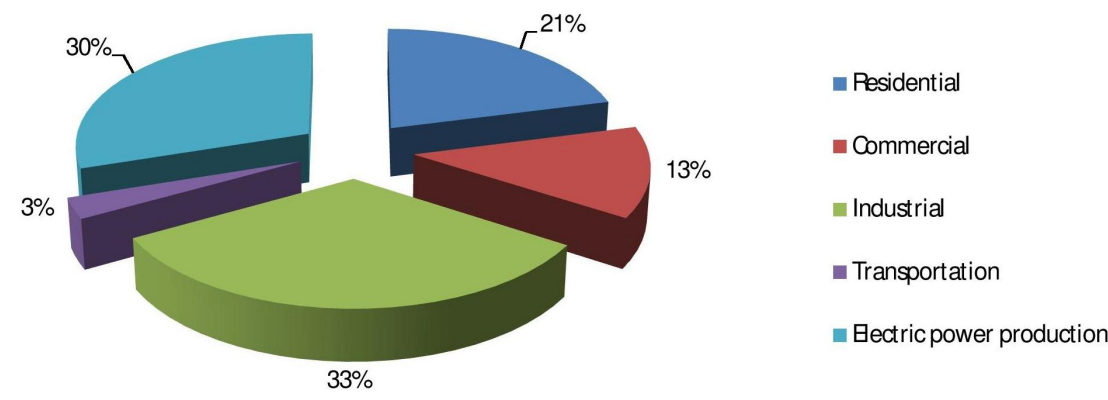

Figure 1.1: Natural Gas Consumption by Sector

NG is delivered to consumers through indirect channels that consist of exploration, extraction, production, transmission, storage and distribution stages. Designing and operating an optimal NG network is important in order to meet customers' demand on time and to minimize costs, especially in transportation stages of transmission and distribution. 
Transmission and distribution systems are the two main components of a NG network. A transmission system can be defined as a high-pressure pipeline system used to transport NG over long distances from suppliers to a distribution centers with large diameter pipelines. Large amounts of NG are transported by compressor stations installed at strategic points along the transmission pipeline.

A distribution system is a lower-pressure pipeline system that takes NG from the transmission system and delivers it to end users including residential, commercial, industrial consumers and power plants. Distribution is provided by local companies that transport gas to customers by small diameter pipelines.

NG transmission pipeline network problems are different from other network flow problems due to the existence of pressure variables and the nonlinear relationship between the pressure drop and flow rate. Gas transmission systems operate at high pressure levels. While gas travels through the pipeline, gas pressure decreases due to friction with the pipe wall. Thus, it is necessary to increase the pressure at a number of points along the pipeline to keep the gas flowing. Compressor stations provide the necessary energy to maintain the required pressure throughout the pipeline. Compressors use electricity or natural gas as an energy source to operate. Many studies focused on minimizing the energy consumption of compressor stations.

With the increasing demand, the size and complexity of NG pipeline networks have also increased. A transmission network may try to expand over time to meet demands at new consumer nodes. The capacity of the system can be increased by adding compressor units to existing stations or by building new stations. The power of the compressor station varies depending on the flow rate.

In the problem, there are many constrains, such as flow conservation, pressure limits and other obstacles. The network should be designed in order to satisfy variable needs over the planning horizon. A well-designed network helps NG companies 
minimize the costs while increasing the customer service level. Thus, a good optimization tool is important to make strategic and operational decisions.

The main thrust of this research is the development of a decision support tool to aid system operators in optimizing NG pipeline operations and the investment costs in order to satisfy customer demand with minimal costs.

\subsection{Research Objectives and Scope}

In NG pipeline networks, design and expansion decisions must be made with careful consideration of the long term benefits. The investment and operating costs, such as installing and operating pipelines and compressor stations, are very high. Compressor station and pipeline installations are part of long-term strategic decisions. Once they are built, they will operate for years. It may cost more than expected to maintain them if these decisions are not made carefully. The aim of this study is to minimize the total investment and operating costs while satisfying the specified requirements corresponding to demands and pressure limits in the system.

The solution to the problem will help to make decisions regarding a new transmission network design, as well as expansion of an existing network, with minimized total cost. In this study, an optimization model is provided to address the following issues:

1. Pressure requirements

2. The best location and capacity of compressor stations that minimizes the cost

3. The best location of pipelines that minimizes the cost

4. The scheduling of installing pipelines and compressor stations in the network

5. The best amount of NG procurement from available suppliers 
While the previous studies in the literature handle different problems of a NG network by using different models, this study proposes an integrated approach to consider these problems in one model. Thus, the aim is to build a mathematical formulation as an advancement to the available studies in the literature.

In all previous studies, the mathematical formulations of pipeline problems include several nonlinear and non-convex constraints and functions. These problems are characterized by a non-convex feasible region, particularly, because of the nonlinear constraint that relates the pressure and the flow rate. The presence of this non-convexity makes the problem hard to solve.

NG transmission network problems can be solved by either heuristic or exact methods. Many studies focused on heuristic methods to solve the nonlinear nonconvex problem. This study proposes utilizing an exact optimization method to solve the problem with a mathematical programming approach.

The organization of this thesis is as follows: In Section 2, a survey of previous related works is introduced. Section 3 explains the NG transmission network problem and the proposed mathematical model. Section 4 presents the solution method and details about the computational study. This is followed by the conclusion and future research direction in Section 5 . 


\section{LITERATURE REVIEW}

In the literature, there are three main network problems which are used to handle different challenges in NG transmission networks.

In NG network design problems, the objective function may be minimization of the investment cost or maximization of the net present value. The output of the model helps to locate the optimal type and the number of compressor stations, and to select the optimal pipe dimensions. Several design variables need to be determined. They include the location and type of compressor stations; possible locations, lengths and diameter of pipelines to be installed; and the allowable operating pressure levels of the system.

NG network flow problems aim to minimize costs and meet demand. Decision variables of the problem are defined to determine gas flow through the pipeline network. The operation cost of NG transmission systems is highly dependent on the compressor station operations because the amount of NG in the system is set by compressor stations. In these problems, selecting the optimal compressor location and capacity is a critical decision.

In network expansion problems, the objective is generally scheduling the investments. To obtain the optimum capacity expansion, investment decisions including time, size and location of pipeline and compressor station installations should be made [14].

This research is based on a comprehensive study that combines three network problems in one model. The main focus is to design a new NG transmission pipeline network or to expand the existing network in order to minimize the investment and operating costs of transporting gas through pipelines in multiple periods. 
Many studies have been done in pipeline network optimization including the pipeline network design, the minimization of fuel consumption of compressor stations, economically locating compressor stations in the network.

Rios-Mercado et al. [24] proposed a reduction technique to minimize the fuel consumption of compressor stations in steady-state transmission networks. This method minimizes the problem dimension at preprocessing without disrupting the problem structure. De Wolf and Smeers [11] modeled the NG pipeline network with nonlinear and linear constraints for a cost minimization problem. They developed a successive linear programming method. The solution procedure is based on piecewise linear approximations of the nonlinear constraint that defines the relationship of the pressure and flow rate. Three test problems with 24, 34 and 60 arcs are solved by an extension of the simplex algorithm. Wu et al. [33] also studied the fuel cost minimization problem. They derived two model relaxations. One relaxation is to develop linear supersets of the non-convex nonlinear compressor domain. The other is to derive piecewise linear functions of the fuel cost objective function. They tested the method by three examples. The first example is a six-node, three-pipe, twocompressor network. The second example is a simple tree network with 10 nodes, 6 pipes, and 3 compressor stations and in the third example there are 48 nodes, 43 pipes, and 8 stations.

Most of the methods developed for this minimization of the fuel consumption problem are based on dynamic programming and gradient search methods. The dynamic programming method was first proposed for a steady state gas transmission system by Wong and Larson [32]. In the study, DP was used to optimize the single source tree-structured network. The objective is to minimize the total compressor energy required to satisfy the specified flow rate, pressure and compressor operation constraints. Dynamic programming guarantees the global optimum. Also, nonlin- 
earity can be easily solved by dynamic programming. However, the implementation of dynamic programming is limited to simple network structures, and computational time increases with the problem size. Two problems were used to represent the performance of the proposed method, one with a single pipeline that has 10 compressors and the other with three single pipelines and a total of 23 compressors.

The study of Borraz-Sanchez and Rios-Mercado [6] aims to find the optimal solution for the compressor station operations in the cyclic NG pipeline network while minimizing the fuel consumption of the stations. The network is represented by pipeline and compressor station arcs and corresponding nodes at the intersection points of the arcs. In the model, there are two continuous decision variables; mass flow rates in each arc and gas pressure at each node. Constraints are non-convex and the objective function is nonlinear. That is, the problem is modeled as a nonlinear program. The proposed solution method is the combination of the non-sequential dynamic programming and the tabu search algorithm. They used various test instances to evaluate the proposed method. The larger problem size has 19 nodes and 7 compressor station arcs.

In other studies, heuristic approaches were proposed in order to minimize compressor station costs. The ant colony optimization algorithm is used for the first time for studying gas flow operations in the study of Chebouba et al. [8]. The main focus of this paper is on using ant colony optimization as a decision tool to obtain fast and accurate results. The objective function of the problem is nonlinear and non-convex. Test instance is composed of one source, one demand and 6 pipelines connected in series by 5 compressor stations. The main interest of the study of RiosMercado et al. [23] is the gas transmission problems with a cyclic tree structure. In this paper a heuristic solution algorithm is proposed. The methodology is composed of two stages. At the first stage, dynamic programming is used to find optimal val- 
ues for pressure variables while the flow variables are fixed. At the second stage, using the optimal value of a pressure variable found at the first stage, a set of flow values, which improve the objective value, are found by a heuristic approach. The proposed method was tested on a tree structured system with 64 nodes, 56 pipes, and 16 compressor stations.

Chung et al. [9] proposed a multi-objective mathematical programming method. Investment costs, reliability and environmental impact compose the three different objectives of the model. They solved the problem by genetic algorithm and adopted a fuzzy decision method to select the best network planning scenario. The model was applied to a network with 13 compressor stations, and 19 pipelines. A hierarchical algorithm is proposed by Hamedi et al. [13] to solve a distribution network problem by using a single-objective, multi-period mixed integer nonlinear programming (MINLP) model. They converted the model into a MIP by adding a set of constraints. The objective is to minimize direct and indirect costs. The model was tested for se seven samples. The smallest test instance include 190 nodes and the largest one has 319 nodes. A MIP model is proposed by Uraikul et al. [28] to optimize the operations of selecting and controlling the compressors. The objective of the study is to minimize the operating costs of the network and meet customer demands in the system. The three factors that affect the costs are the capacities of compressors, the energy used to turn on the compressors, and the energy used to turn them off. The model was tested on a network that has two compressor stations, two customer locations and six periods.

Kabirian and Hemmati [15] developed an integrated nonlinear optimization model for formulating a strategic plan to find the best long-run development plans for an existing network. A heuristic random search optimization method is proposed to solve the problem. The objective is to minimize the net present value of operating 
and investment costs. They used a network with 2 compressor stations, 4 demand, 3 supply and 1 transshipment nodes, and 10 pipelines to assess the performance of the model.Pratt and Wilson [22] propose a mixed integer linear programming (MIP) method to solve the nonlinear optimization problem iteratively. They linearized the pressure drop-flow equation and used the branch-and-bound (BB) algorithm to solve the problem. Osiadacz and Bell [21] suggest a simplified algorithm for the transientstate gas transmission network to find the maximum feasible outlet pressure level of a station. They studied a large-scale network with several compressor stations and they solved the problem by local optimization.

Woldeyohannes and Majid [31] developed a simulation model by incorporating compressor station parameters including speed, suction and discharge pressure. The model is used to simulate the transmission pipeline network system under various conditions to determine pressure and flow parameters. The proposed simulation model in this study could be used to assist in operational and design decisions.

The review of papers in the scope of optimization in NG transmission network based on the decisions made are classified in Table 2.1. Table 2.2 is a summary of the NG network optimization problems. 
Table 2.1: Classification of Natural Gas Network Planning Literature

\begin{tabular}{|c|c|c|c|c|c|c|c|c|}
\hline \multirow[b]{2}{*}{ Author } & \multicolumn{2}{|c|}{ Network Design } & \multicolumn{4}{|c|}{ Network Flow and Operation } & \multicolumn{2}{|c|}{ Network Expansion } \\
\hline & $\begin{array}{r}\text { Min. } \\
\text { Invest } \\
\text { Cost }\end{array}$ & $\begin{array}{l}\text { Max. } \\
\text { NPV }\end{array}$ & $\begin{array}{c}\text { Min. } \\
\text { Transprt. } \\
\text { Cost }\end{array}$ & $\begin{array}{l}\text { Compr. } \\
\text { Select. to } \\
\text { Min.Costs }\end{array}$ & $\begin{array}{l}\text { Min. } \\
\text { Fuel } \\
\text { Cost }\end{array}$ & $\begin{array}{c}\text { Min. } \\
\text { Supply } \\
\text { Cost }\end{array}$ & $\begin{array}{c}\text { Opt. } \\
\text { Investment } \\
\text { Scheduling }\end{array}$ & $\begin{array}{c}\text { Min. } \\
\text { Invest. } \\
\text { Cost }\end{array}$ \\
\hline Wu et al. [33] & - & - & - & - & $\checkmark$ & - & - & - \\
\hline Uraikul et al. [28] & $\checkmark$ & - & - & $\checkmark$ & - & - & - & - \\
\hline Chung et al. [9] & - & - & - & - & - & - & $\checkmark$ & - \\
\hline Rios-Mercado et al. [23] & - & - & - & - & $\checkmark$ & - & - & - \\
\hline Kabirian et al..[15] & $\checkmark$ & - & - & - & - & - & $\checkmark$ & - \\
\hline Borraz-Sanchez et al. [6] & - & - & - & - & $\checkmark$ & - & - & - \\
\hline Hamedi et al. [13] & - & - & $\checkmark$ & - & - & - & - & - \\
\hline De Wolf and Smeers [11] & - & - & - & - & - & $\checkmark$ & - & - \\
\hline Chebouba et al. [8] & - & - & - & $\checkmark$ & $\checkmark$ & - & - & - \\
\hline Wong and Larson [32] & - & - & - & $\checkmark$ & $\checkmark$ & - & - & - \\
\hline This Study & $\checkmark$ & - & $\checkmark$ & - & - & $\checkmark$ & $\checkmark$ & $\checkmark$ \\
\hline
\end{tabular}

Table 2.2: Characteristics of Natural Gas Optimization Problems

\begin{tabular}{|c|c|c|c|c|c|c|c|c|c|}
\hline & & \multicolumn{2}{|c|}{ System } & \multicolumn{2}{|c|}{ Topology } & \multicolumn{2}{|c|}{$\begin{array}{l}\text { Solution } \\
\text { Method }\end{array}$} & \multicolumn{2}{|c|}{$\begin{array}{c}\text { Number of } \\
\text { Periods }\end{array}$} \\
\hline Author & Model & State & Transient & Cyclic & Tree & Exact & Heuristic & Single & Multi \\
\hline Wu et al. [33] & NLP & $\checkmark$ & - & - & - & - & $\checkmark$ & $\checkmark$ & - \\
\hline Uraikul et al. [28] & MILP & $\checkmark$ & - & - & - & $\checkmark$ & - & - & $\checkmark$ \\
\hline Chung et al. [9] & NLP & - & - & - & - & - & $\checkmark$ & $\checkmark$ & - \\
\hline Rios-Mercado et al. [23] & NLP & - & - & $\checkmark$ & - & - & $\checkmark$ & $\checkmark$ & - \\
\hline Kabirian et al.[15] & NLP & $\checkmark$ & - & - & - & - & $\checkmark$ & - & $\checkmark$ \\
\hline Borraz-Sanchez et al. [6] & NLP & $\checkmark$ & - & $\checkmark$ & - & - & $\checkmark$ & $\checkmark$ & - \\
\hline Hamedi et al. [13] & NLP & $\checkmark$ & - & - & - & $\checkmark$ & $\checkmark$ & - & $\checkmark$ \\
\hline De Wolf and Smeers [11] & NLP & $\checkmark$ & - & - & $\checkmark$ & - & $\checkmark$ & $\checkmark$ & - \\
\hline Chebouba et al. [8] & NLP & $\checkmark$ & - & - & $\checkmark$ & - & $\checkmark$ & $\checkmark$ & - \\
\hline Wong and Larson [32] & NLP & $\checkmark$ & - & - & $\checkmark$ & $\checkmark$ & - & $\checkmark$ & - \\
\hline This Study & MINLP & $\checkmark$ & - & - & $\checkmark$ & $\checkmark$ & - & - & $\checkmark$ \\
\hline
\end{tabular}




\section{PROBLEM DEFINITION AND MATHEMATICAL FORMULATION}

A typical NG pipeline network problem consists of demand and supply nodes, pipelines, and compressor stations. In such complex and large networks, proper planning is important because even a small reduction in investment and operation expenses provides considerable amounts of saving. NG networks continue to grow with the increasing demand and this growth makes the network more complex. Thus, developing effective solution methods becomes more important.

An optimization approach is proposed to solve the problem of how to optimally design the network and operate the gas flow in the pipeline system with deterministic parameters. In this study, it is assumed that the transmission system, consisting of multiple suppliers and multiple consumers, is operated only by the NG Company.

\subsection{Characteristics of the System}

There are two different states in the gas network depending on the gas flow-time relationship. If a system is in a steady state, then gas flow through the system is independent of time. These systems can be modeled by algebraic nonlinear equations. In a transient state system, gas flow changes in time, thus, partial differential equations are required to describe this relation. In this research, a steady-state gas transmission network system will be studied.

Another characteristic of transmission systems is the topology of the network. There are two main structure types of gas networks. A cyclic topology is a network with at least one cycle. A tree structured (non-cyclic) topology is a network that does not contain any cycles. These networks may contain a number of different trees. The main focus of the study will be on the transmission of gas through a tree-structured pipeline network system. 
A NG transmission network studied in this research consists of demand, supply, transshipment, and compressor station inlet and outlet nodes. In the network, pipelines and compressor stations are represented by directed arcs. Demand nodes are the locations of NG consumer cities. Supply nodes are the sources of gas. Transshipment nodes are the connection points of two or more arcs. In transshipment nodes, incoming flow is equal to outgoing flow. Compressor station inlet nodes are the points where gas enters a station. Compressor station outlet nodes are the end points of compressor station arcs where compressed gas exits a station. The impacts of other elements including valves and regulators are negligible for this study.

Figure 3.1 show the graphical notation of the network.

\begin{tabular}{ll}
$\square$ & Supply Node \\
$\bigcirc$ & Demand Node \\
$\longrightarrow$ & Transshipment Node \\
& Active Pipe Arcs \\
\hdashline$\quad$ Inactive Pipe Arcs \\
$\square \quad$ Closed Compressor Station \\
$\square \rightarrow \square \quad$ Bypassed Compressor Station \\
$\square \rightarrow \square \quad$ Active Compressor Station
\end{tabular}

Figure 3.1: Graphical Notation for the Networks 


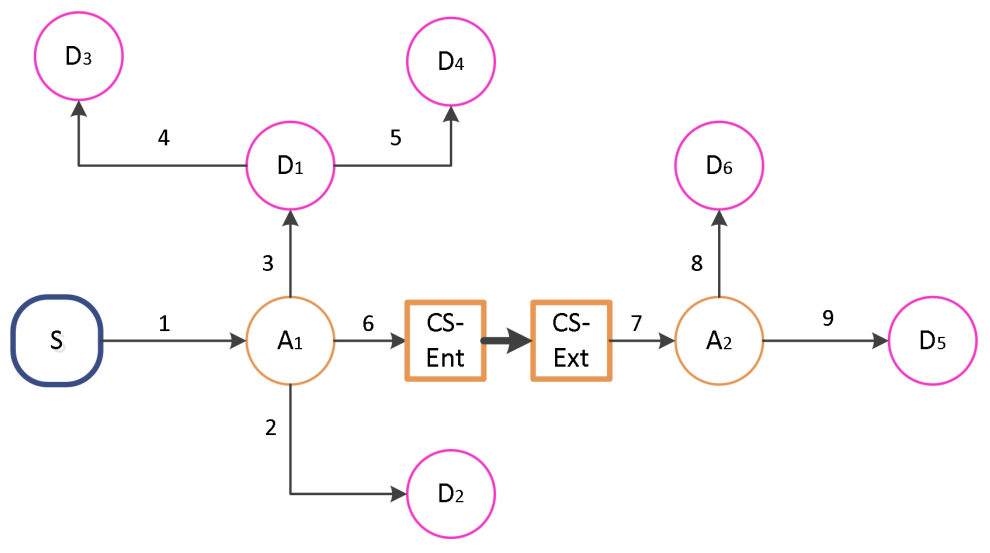

Figure 3.2: A Natural Gas Transmission Pipeline Network

A typical network of the problem with 11 nodes, 9 pipe arcs, and 1 compressor arc is shown in Figure 3.2. Node $\mathrm{S}$ is a supply node, where gas is purchased from. Node D1, D2, D3, D4, D5, and D6 are demand nodes, where gas is consumed. Node A is a transmission node. Nodes CS-Ent and CS-Ext are compressor station inlet and outlet nodes, respectively.

In the proposed model, there are several decision variables related to the components of the system. The positive continuous variables are pressures at nodes, gas flow rates in pipelines and the supply amount. The sets of binary variables are used to define the flow directions, compressor station locations and types, and pipeline locations.

The objective is to minimize the total investment and operating cost of the network. Investment costs are the installation costs of pipelines and compressor stations. Operating costs consist of transporting cost, operation cost of compressor stations and pipelines, such as maintenance, energy, etc., and purchase cost of supply. The costs are varied for each period so the total cost is the sum of periodic costs. 
The mathematical model of the problem is a MINLP, where the objective function is linear and the set of constraints including linear and nonlinear inequalities with binary and continuous variables. The model includes various linear constraints for mass flow conservation for supply, demand and transshipment nodes. Pressureflow rate relation will be defined by nonlinear inequalities. Moreover, there will be constraints related to whether compressor station existence and to its capacity.

The problem is described as a multi-period network problem model in order to allow making changes in the network over the planning horizon. These changes can be exogenous, i.e. the existence of new demand nodes, in response to increasing demand. As a result of these, new endogenous changes may be needed such as adding new pipelines, and compressor stations. It is assumed that these changes are long-lasting, which means once a new pipeline/compressor station is installed, or a new demand node is added to the network, then it is available during all planning horizons in the network.

\subsection{System Components}

\subsubsection{Pipelines}

The relationship between the flow rate and the pressure, and the definition of pressure values as state variables at nodes, are the major characteristics of the transmission network in steady-state. Flow rate is a function of the pressure difference across the pipe, the diameter and length of the pipe, and properties of gas. Using the same function, the pressure values can be determined by flow rate and pipeline resistance.

The properties of pipelines and gas are important to determine the pipeline resistance so the pipeline resistance determines the pressure drop. While gas flows, pressure decreases due to pipeline resistance and flow losses. At every demand node, 
the amount of flow in the pipeline decreases as well as the pressure. The pressure difference between the end nodes of a pipeline depends on the resistance.

Several variations of the general flow equation have been proposed to calculate the gas flow rate in a pipeline, such as the Weymouth equation, the Panhandle A and Panhandle B equations [20]. These equations differ from each other by the system size range they can be applied to and the treatment of pipe friction. The General Flow Equation for the steady-state flow in a gas pipeline is the basic equation for relating the pressure drop to flow rate. In the system, gas flows at a constant temperature (isothermal flow) through a horizontal pipe segment. The pipe segments are assumed to be long enough so that kinetic energy changes can be negligible [16]. Figure 3.3 shows a steady flow in the gas pipeline.

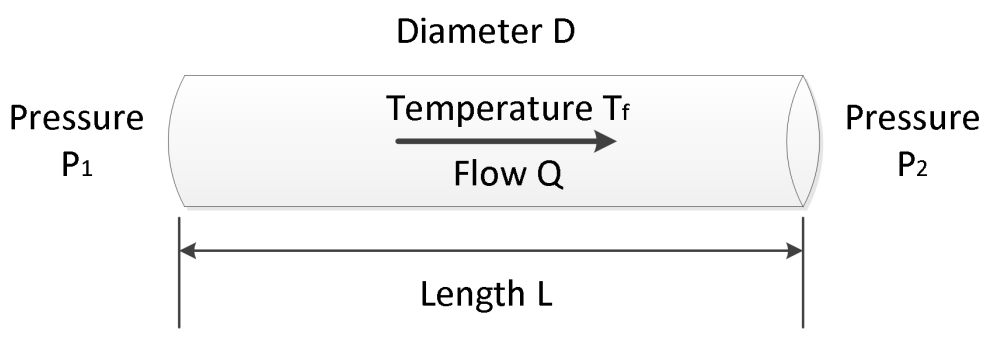

Figure 3.3: Steady Flow in Gas Pipeline

In The International System of Units (SI) units, the General Flow equation is stated as follows:

$$
Q_{i j}=1.149410^{-3}\left(\frac{T_{b}}{P_{b}}\right)^{2}\left[\frac{P_{i}^{2}-P_{j}^{2}}{G T_{f} L_{i j} Z f}\right]^{0.5} D_{i j}^{2.5}
$$

where friction factor $(f)$, base pressure $\left(P_{b}\right)$ and temperature $\left(T_{b}\right)$, gas gravity $(G)$, average gas flowing temperature $\left(T_{f}\right)$, gas compressibility factor $(Z)$ are assumed to 
be constants. This equation can be applied where fully turbulent gas flow under high pressure is in question [20].

Equation relates the flow rate in a pipe with a length $L(\mathrm{~km})$ and a diameter $D$ $(\mathrm{mm})$, based on an upstream pressure $P_{i}$ (bar) and a downstream pressure $P_{j}$ (bar). The flow rate $Q\left(\mathrm{~m}^{3} /\right.$ day $)$ depends on gas properties such as the gravity $G$ (0.66 for $\mathrm{NG}$ ) and the compressibility factor $Z$ (0.805 for NG). The pressure drop, from the upstream point $i$ to downstream point $j$, occurs due to friction between the gas and pipe walls with a typical friction factor $f$ (generally 0.01 ). The pressure difference of the two nodes, the upstream end and the downstream end, determines the direction of the gas flow. When there is no flow rate between nodes $i$ and $j, P_{i}$ is equal to $P_{j}$. Since the volume of gas changes according to the ambient temperature and pressure, base temperature $T_{b}\left({ }^{\circ} \mathrm{K}\right)$ and pressure $P_{b}$ (bar) are necessary to provide standard conditions for gas measurement. In this study, base temperature $T_{b}$ is $288^{\circ} \mathrm{K}$ and base pressure is $P_{b}$ is 1 bar while the average gas flowing temperature $T_{f}$ is $283^{\circ} \mathrm{K}$.

It is assumed that the unit of flow rate $Q$ is million cubic meters (mmscm), so all calculations in this study are made accordingly. The studied transmission network is composed of horizontal pipelines. In transmission networks, pipe sizes generally vary between 12 and 48 inches in diameter, 5 and $100 \mathrm{~km}$ in length. In this study, the diameter of pipelines are assumed to be fixed to 30 inches, but the lengths are variable. In The pipe flow equation that relates the pressure drop to the flow rate of high pressure flows in a steady state is represented as

$$
P_{i t}^{2}-P_{j t}^{2}=\delta_{i j} Q_{i j t}^{2} \quad \forall(i, j) \in A_{P}
$$

where the value of $\delta_{i j}$ (pipeline resistance) depends on the properties of gas and also the dimensions of the pipe $(i, j)$. 
In this study, the general flow equation is used to calculate the pressure drop. Thus, the pipeline resistance can be expressed as

$$
\delta_{i j}=0.756910^{6} \frac{G T_{f} L_{i j} Z f}{D_{i j}^{5}}\left(\frac{P_{b}}{T_{b}}\right)^{2} \quad \forall(i, j) \in A_{P}
$$

As mentioned earlier, pressure drop and flow rate depend on pipeline properties. Thus, it is often necessary to decide pipe sizes before calculating pressure drop and flow rate. The pressure levels at nodes can be calculated, by knowing the flow rate and available pipe sizes in advance. Then, the compressor stations can be located at necessary points.

As the pipe length increases for a given flow rate, the pipeline resistance increases as well as the pressure drop. For pipes of the same diameter, the pressure difference is greater between two ends of a long one than a shorter one. On the other hand, a pipe with a large diameter has less resistance than a pipe with a smaller diameter. Thus, the pressure drop is smaller across a pipe with a large diameter [20].

\subsubsection{Compressor Stations}

Compressor stations are one of the most important assets in transmission pipeline network systems worldwide. They are installed to provide the pressure needed to transport gas through pipelines. They can be defined simply as a device to compress gas molecules in order to provide enough energy to keep it moving along the transmission line. Due to the limitations of pipeline pressures, multiple compressor stations may be needed to transport a given volume through a long-distance pipeline. The pressures at which these compressor stations operate are determined by the pipe pressure levels and the power available [20]. In this study, the set of nodes representing compressor stations consists of inlet (CS-Ent) and outlet (CS-Ext) nodes. 
If there is not any flow between the two nodes then these nodes are treated as one node. Figure 3.4 shows the representation of nodes and arcs in the system.

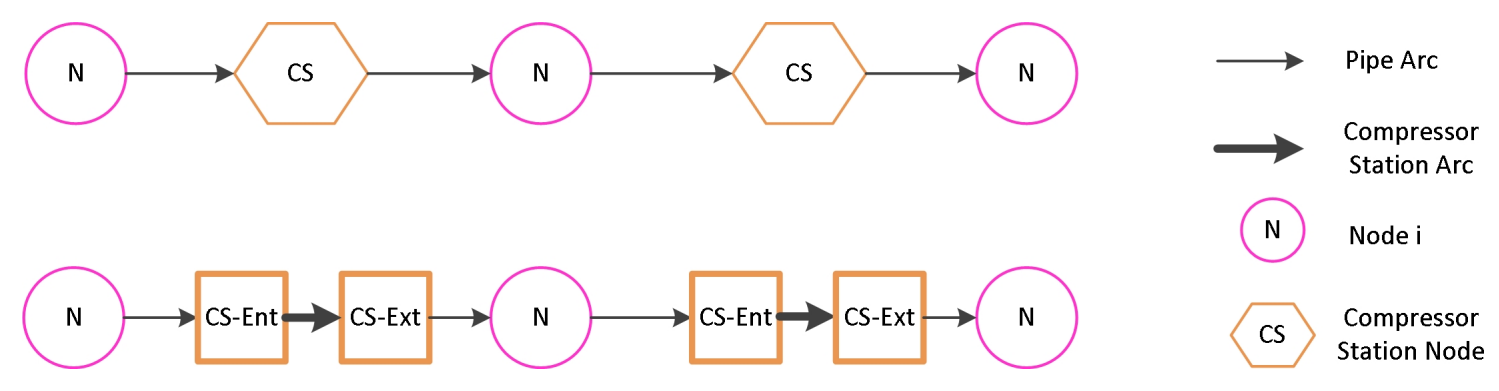

Figure 3.4: Representation of Nodes and Arcs

In general, gas leaves the station at the discharge pressure of 75 bar. As the gas flows, pressure in the pipeline decreases due to friction and flow losses. If the pressure in the pipeline is below the allowable pressure level at some point, then a compressor station is required, otherwise gas flow will cease. Compressor stations increase the pressure to a certain level. Therefore, while gas flows from the upstream node to the downstream node in pipeline arcs, it flows in the opposite direction in compressor station arcs. Gas pressure cannot be greater than the allowable operating pressure of the pipeline. There is no flow loss due to friction in the compressor arcs. Figure 3.5 shows the changes in the flow rate and the pressure through pipe and compressor arcs.

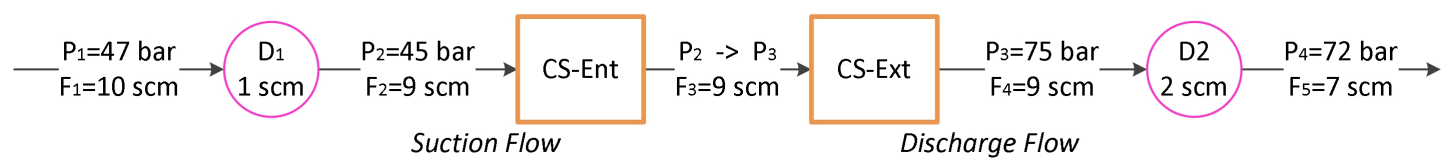

Figure 3.5: Representation of Compressor Station 
A compressor station may be active, bypassed or closed. These states will be decided according to the solution of the problem. If the outlet pressure value is greater than the inlet pressure value at the compressor nodes, then the station is active. When the outlet and inlet pressure values are equal, the station is bypassed. If there is no flow through the compressor arc, then the compressor station is closed.

While designing a pipeline system, the possible locations and sizes of the compressor stations must be determined. The size of a compressor station, or, more precisely the number of units that must be installed, depends on the mass flow rate. In this study, it is assumed that compressor units installed in a station are identical, and the maximum flow rate between two compressor station nodes is $20 \mathrm{mmscm} /$ day. The number of stations in the system depends on the length of the transmission pipeline. More stations are required if the length of the line increases. Environmental and geotechnical factors are important in selecting the station location.

\subsubsection{Cost Structure}

In pipeline network problems, generally, the cost function consists of investment and operating costs. The major components of the gas transmission system that account for the investment costs are the pipeline and compressor stations. These costs constitute the important part of the total pipeline project cost. Operating costs, such as maintenance, energy, transmission, utility, as well as general and administrative, are the recurring periodic costs.

The total cost for gas transmission pipeline network can be calculated as follows;

$$
\begin{aligned}
\text { Total Cost } & =[\text { investment cost }+ \text { operating cost }]_{\text {pipeline }} \\
& +[\text { investment cost }+ \text { operating cost }]_{\text {compressor station }} \\
& + \text { natural gas purchase cost }+ \text { transportation cost }
\end{aligned}
$$


The cost parameters and their units are shown in Table 3.1 [20].

Table 3.1: Data for Cost Computation

\begin{tabular}{|c|c|c|c|}
\hline Parameter & Definition & Value & Unit \\
\hline$Q$ & daily flow rate in a compressor station arc & 20 & $\mathrm{mmscm} /$ day \\
\hline$T$ & average gas flowing temperature & 283 & $\mathrm{~K}$ \\
\hline$Z$ & gas compressibility factor at the flowing temperature & 0.805 & dimensionless \\
\hline$f$ & friction factor & 0.01 & dimensionless \\
\hline$G$ & gas gravity & 0.66 & dimensionless \\
\hline$P_{2}$ & outlet pressure of a compressor station & 75 & bar \\
\hline$P_{1}$ & inlet pressure of a compressor station & 45 & bar \\
\hline$T_{b}$ & base temperature & 288 & K \\
\hline$P_{b}$ & base pressure & 1 & bar \\
\hline$E_{p}$ & polytropic efficiency & 0.9 & decimal value \\
\hline$b_{l}$ & bearing losses & 0.02237 & bar \\
\hline$s_{l}$ & seal losses & 0.0149 & bar \\
\hline$i$ & interest rate & 0.12 & dimensionless \\
\hline$k$ & ratio of specific heats of gas & 1.24 & dimensionless \\
\hline$R p$ & $\begin{array}{l}\text { fraction between pipe installation cost } \\
\text { and the pipe price itself }\end{array}$ & 1.4 & dimensionless \\
\hline$l$ & $\begin{array}{l}\text { nonlinear constant between pipe's price } \\
\text { and pipe length }\end{array}$ & 1 & dimensionless \\
\hline$m$ & $\begin{array}{l}\text { nonlinear constant between pipe's price } \\
\text { and pipe diameter }\end{array}$ & 1 & dimensionless \\
\hline$b$ & $\begin{array}{l}\text { nonlinear constant between compressor price } \\
\text { and its power }\end{array}$ & 0.8 & dimensionless \\
\hline$C_{o p}$ & $\begin{array}{l}\text { fraction of compressor operating cost } \\
\text { (excluding energy cost) }\end{array}$ & 0.75 & dimensionless \\
\hline$C_{f p}$ & $\begin{array}{l}\text { fraction of pipe operating cost to } \\
\text { the annual investment cost of pipeline }\end{array}$ & 0.2 & dimensionless \\
\hline$C_{e c}$ & electricity price & 75 & $\mathrm{US} \$ / \mathrm{MWh}$ \\
\hline$C_{p}$ & pipe unit price & 1,870 & $\mathrm{US} \$ / \mathrm{km} /$ inch \\
\hline$C_{m w}$ & compressor price & $2,000,000$ & $\mathrm{US} \$ / \mathrm{MW}$ \\
\hline
\end{tabular}

All investment costs are converted to annualized costs for the useful life of the assets. In this study, the straight line depreciation method is used to calculate the annual investment costs. Straight line depreciation is calculated as follows;

$$
\text { Depreciation }=\frac{(\text { Purchase Price of Asset }- \text { Approximate Salvage Value })}{\text { Estimated Useful Life of Asset }}
$$


It is assumed that the entire life of the system is 45 years and the salvage values of the assets are zero at the end of the life of the assets. The net present values of the annual investments in the first 12 years are computed based on a $12 \%$ interest rate. Net present value is expressed as

$$
N P V=C_{0}-\sum_{t=1}^{T} \frac{C_{t}}{(1+i)^{t}}
$$

\subsubsection{INVESTMENT COST FOR PIPELINE}

The investment cost of the pipeline including the material, labor, installation, and right of way costs, depends on pipe length and diameter [1] . It can be expressed as

$$
\alpha_{t}^{p}=\left(1+R_{p}\right) C_{p} \text { length }^{l} \text { dia }^{m}
$$

The value of $C p, l, m$ can be found easily by regression if the price of pipe is known. In this study, they are assumed to be 1 .

\subsubsection{INVESTMENT COST FOR COMPRESSOR STATIONS}

To build the objective function, it is assumed that electricity is used to operate the compressor stations. Tax, insurance, and other costs are not included in the cost function. The fixed compressor station cost changes depending on the installed power (MW). The following is the expression for compressor power [1] .

$$
g_{m w}=3.0325 \frac{Q P_{b} T Z\left[\left(\frac{P_{2}}{P_{1}}\right)^{\left(\frac{k-1}{k E_{p}}\right)}-1\right] k}{T_{b}(k-1)}+b_{l}+s_{l}
$$


where $Q$ is the daily flow rate in a compressor station arc. The investment cost of compressor stations is computed as follows

$$
\alpha_{t}^{c}=C_{m w}\left(g_{m w}\right)^{b}
$$

where $C_{m w}$ is a overall cost of material, equipment, labor, right of way per $1 \mathrm{MW}$. $C_{m w}$ and $b$ values can be found by regression as well.

By using the given parameters, the power of each compressor station unit is 10 MW.

\subsubsection{PIPELINE OPERATING COST}

The pipeline operating cost is the cost of maintaining pipes. It is assumed the operating cost is proportional to the annual investment cost [1]. For each pipeline segment the operating cost is computed as follows;

$$
\beta_{t}^{p}=C_{f p} \alpha_{t}^{p}
$$

\subsubsection{COMPRESSOR STATION OPERATING COST}

The operating cost of a compressor station is electricity and maintenance cost. The operating cost can be formulated as a proportional to the electricity cost [1] . Thus, it is expressed as follows;

$$
\beta_{t}^{c}=\left(1+C_{o p}\right) C_{e c}
$$

To calculate the electricity cost, the unit of compressor power is converted into megawatt-hour, then 


$$
g_{M W h}=19.809 \frac{Q P_{b} T Z\left[\left(\frac{P_{2}}{P_{1}}\right)^{\left(\frac{k-1}{k E_{p}}\right)}-1\right] k}{T_{b}(k-1)}+6.532(b l+s l)
$$

and the electricity cost is

$$
C_{e c}=g_{M W h} C_{e}
$$

At the beginning of the planning horizon the electricity price is $75 \$ / M W h$. It is assumed that the electricity price increases $5 \%$ each year.

Table 3.2 shows periodic investment and operating costs of pipelines and compressor stations.

Table 3.2: Investment and Operating Costs $\left(10^{3} \$\right)$

\begin{tabular}{|c|c|c||c|c|c||c|c|c||c|c|c|}
\hline Cost & Period & Actual Cost & Cost & Period & Actual Cost & Cost & Period & Actual Cost & Cost & Period & Actual Cost \\
\hline \multirow{5}{*}{} & 1 & 10243.865 & & 1 & 24.71 & & 1 & 109.30 & & 1 & 21.86 \\
& 2 & 9878.22 & & 2 & 25.94 & & 2 & 105.4 & & 2 & 21.08 \\
& 3 & 9459.01 & & 3 & 27.24 & & 3 & 100.92 & & 3 & 20.19 \\
& 4 & 8978.67 & & 4 & 28.6 & & 4 & 95.8 & & 4 & 19.16 \\
& 5 & 8428.56 & & 5 & 30.03 & & 5 & 89.93 & & 5 & 17.99 \\
& 6 & 7798.84 & \multirow{3}{*}{$\beta_{t}^{c}$} & 6 & 31.54 & & 6 & 83.21 & & 6 & 16.64 \\
& 7 & 7078.33 & & 7 & 33.12 & $\alpha_{t}^{p}$ & 7 & 75.52 & $\beta_{t}^{p}$ & 7 & 15.1 \\
& 8 & 6254.31 & & 8 & 34.77 & & 8 & 66.73 & & 8 & 13.35 \\
& 9 & 5312.31 & & 9 & 36.51 & & 9 & 56.68 & & 9 & 11.34 \\
& 10 & 4235.89 & & 10 & 38.33 & & 10 & 45.2 & & 10 & 9.04 \\
& 11 & 3006.35 & & 11 & 40.25 & & 11 & 32.08 & & 11 & 6.42 \\
& 12 & 1602.43 & & 12 & 42.26 & & 12 & 17.1 & & 12 & 3.42 \\
\hline
\end{tabular}

\subsubsection{NG PURCHASE COST}

In real world systems, the supply contracts include requirements for pressure levels at inlet nodes, periodic amount, and prices. There might be multiple suppliers in the system, and each of them might have different regulations about exporting gas. In the study, purchase cost is the charge per mmscm of gas purchased and it may 
vary for each supplier [1]. Gas purchase cost is assumed to increase $2 \%$ each year. Table 3.3 shows purchase cost of each supplier in each period.

Table 3.3: Purchase Costs $\left(10^{3} \$\right)$

\begin{tabular}{|c|c|c|c|c|}
\hline Period & Supplier 1 & Supplier 2 & Supplier 3 & Supplier 4 \\
\hline 1 & 250.00 & 300.00 & 350.00 & 275 \\
2 & 255.00 & 306.00 & 357.00 & 280.50 \\
3 & 260.10 & 312.12 & 364.14 & 286.11 \\
4 & 265.30 & 318.36 & 371.42 & 291.83 \\
5 & 270.61 & 324.73 & 378.85 & 297.67 \\
6 & 276.02 & 331.22 & 386.43 & 303.62 \\
7 & 281.54 & 337.85 & 394.16 & 309.70 \\
8 & 287.17 & 344.61 & 402.04 & 315.89 \\
9 & 292.92 & 351.50 & 410.08 & 322.20 \\
10 & 298.77 & 358.53 & 418.28 & 328.65 \\
11 & 304.75 & 365.70 & 426.65 & 335.22 \\
12 & 310.84 & 373.01 & 435.18 & 341.93 \\
\hline
\end{tabular}

\subsubsection{TRANSPORTATION COST}

Since transportation is a major component of the total cost of the system, many studies on transmission pipeline properties are promoted in order to find an optimum system design for economical transmission. For every unit of gas transported, the pipeline owner pays the transportation cost to maintain the transportation. The transportation cost can be defined as the cost of service to transport a unit of gas through a segment of pipeline. The transportation cost is charged per mmscm of gas. It is expressed as

$$
\beta_{t}^{s}=\frac{\left(\sum_{i, j} \text { length }_{i j}\left(\beta_{t}^{p}+\alpha_{t}^{p}\right)\right)+\beta_{t}^{c}+\alpha_{t}^{c}}{F_{i j t}}
$$


where $\overline{F_{i j t}}$ is the average annual flow rate [1]. It is assumed that the average annual flow rate is $7000 \mathrm{mmscm}$. For The total pipeline length in 31-node is network 1040 $\mathrm{km}$, in 66 -node it is $2440 \mathrm{~km}$, and in 97 -node it is 3500 . Table 3.4 shows transportation cost of each period.

Table 3.4: Transportation Costs $\left(10^{3} \$\right)$

\begin{tabular}{|c|c|c|c|c|c|c|c|c|}
\hline Network & Period & $\beta_{t}^{s}$ & Network & Period & $\beta_{t}^{s}$ & Network & Period & $\beta_{t}^{s}$ \\
\hline \multirow{12}{*}{ 31-node } & 1 & 20.95 & \multirow{12}{*}{ 66-node } & 1 & 47.18 & \multirow{12}{*}{ 97-node } & 1 & 67.05 \\
\hline & 2 & 20.21 & & 2 & 45.50 & & 2 & 64.65 \\
\hline & 3 & 19.35 & & 3 & 43.57 & & 3 & 61.91 \\
\hline & 4 & 18.37 & & 4 & 41.36 & & 4 & 58.77 \\
\hline & 5 & 17.24 & & 5 & 38.82 & & 5 & 55.17 \\
\hline & 6 & 15.95 & & 6 & 35.92 & & 6 & 51.04 \\
\hline & 7 & 14.48 & & 7 & 32.61 & & 7 & 46.33 \\
\hline & 8 & 12.80 & & 8 & 28.81 & & 8 & 40.94 \\
\hline & 9 & 10.87 & & 9 & 24.47 & & 9 & 34.77 \\
\hline & 10 & 8.67 & & 10 & 19.51 & & 10 & 27.73 \\
\hline & 11 & 6.15 & & 11 & 13.85 & & 11 & 19.68 \\
\hline & 12 & 3.28 & & 12 & 7.39 & & 12 & 10.49 \\
\hline
\end{tabular}

\subsection{Modeling Assumptions}

The NG transmission network is characterized as a complex system so several assumptions are required to simplify the expression of the problem by the mathematical programming model and to limit the scope of the study.

This study focused on a gas transmission pipeline network with large diameter pipelines that transport a large amount of gas at high pressures over long distances. It is assumed that the problem is in steady state, thus algebraic equations can be used to describe the flow of NG through the network. The mathematical model provides solutions for each period that has variable demand. The model is deterministic so 
each parameter is assumed to be known in advance. The model is built on a cyclic network, but the output of the model is a tree network. Therefore, there are no loops in the output.

Since a gas transmission network problem is also a network flow problem, mass flow conservation must be satisfied in the system. The key characteristic of the problem is the presence of pressure variables. The allowed maximum pressure depends on a Gas Company's needs. In the network, pressure requirements will be met and NG will be forced to flow in one direction per period.

\subsection{Network Structure}

The transmission pipeline network is modeled in a directed graph $G=(N, A)$. $N$ is a finite set of nodes; $i \in\{1,2, \ldots,|N|\}$ and it consists of supply $\left(N_{s}\right)$, demand $\left(N_{d}\right)$, transshipment $\left(N_{t}\right)$, compressor station inlet and outlet nodes $\left(N_{c}\right)$. The set of nodes can be defined as $N=N_{s} \cup N_{d} \cup N_{t} \cup N_{c}$. A is a set of arcs, which are the ordered pairs $(i, j)$ of distinct nodes in $N . A$ is a union set of pipe and compressor station arcs in the network, i.e., $A=A_{p} \cup A_{c}$, with $A_{p} \cap A_{c}=\emptyset$. If $(i, j) \in A_{c}$ then $i, j \in N_{c}$ are the nodes representing the inlet and outlet nodes of a compressor station. A similar interpretation can be made for pipeline arcs $(i, j) \in A_{p}$ where $i, j \in N$.

Pipeline arcs function with different gas flows between two nodes to satisfy changing customer demands over multi-period. The network must contain at least one pipe arc connected to a demand node. Since the network is described as a directed graph, back-flow in the pipes is not allowed. Pressure decreases along the pipe arcs, while it increases along the compressor station arcs. In the following, the problem is formulated as a MINLP model. 


\subsection{The Mathematical Model}

The mathematical formulation of the gas transmission network problem is given as follows;

NOTATIONS:

Sets:

$T \quad$ set of periods

$U$ set of the number of compressor stations units

$N_{s} \quad$ set of supply nodes

$N_{d} \quad$ set of demand nodes

$N_{t} \quad$ set of transshipment nodes

$N_{c} \quad$ compressor station nodes

$N \quad$ the union of sets $N_{s}, N_{d}, N_{t}$, and $N_{c}$

$A_{c} \quad$ set of compressor station arcs

$A_{p} \quad$ set of pipeline arcs

$E \quad$ the union of sets $A_{c}$ and $A_{p}$

\section{Parameters:}

$\theta_{i} \quad$ demand of $i^{\text {th }}$ node in period $t \in T$

$\delta_{i j} \quad$ pipeline resistance between nodes $(i, j) \in A_{p}$

$\alpha_{t}^{c} \quad$ investment cost of installing one unit of a compressor station in period $t \in T$

$\alpha_{t}^{p} \quad$ investment cost of installing a pipeline in period $t \in T$

$\beta_{t}^{c} \quad$ operating cost of one unit of a compressor station in period $t \in T$

$\beta_{t}^{p} \quad$ operating cost of one $\mathrm{km}$ of a pipeline arc in period $t \in T$

$\beta_{t}^{s} \quad$ transportation cost $t \in T$

$\beta_{i t}^{f} \quad$ NG purchase cost of supplier $i \in N_{s} t \in T$

$\sigma \quad$ capacity of one unit of a compressor station

$\phi_{i j} \quad$ length of available pipelines $(i, j) \in A_{p}$ 


\section{Decision Variables:}

$P_{i t} \quad$ pressure at node $i$ in period $t \in T$

$Q_{i j t} \quad$ mass flow rate between nodes $i$ and $j$ in period $t \in T$

$B_{i j t} \quad 1$ if gas flows from $i$ to $j$ in period $t \in T, 0$ otherwise

$C S_{i j u t} \quad 1$ if a compressor station is installed at nodes $(i, j) \in N_{c}$ with $u$ units in period $t \in T, 0$ otherwise

$V_{i j t} \quad 1$ if a new pipeline is installed between nodes $i$ and $j$ in period $t \in T$, 0 otherwise

$G_{i t} \quad 1$ if the pressure is below 45 bar at node $i \in N_{c}$ in period $t \in T$, 0 otherwise

MODEL:

$$
\begin{aligned}
& \operatorname{Min} \sum_{(i, j) \in A_{p}} \sum_{t \in T} Q_{i j t} \beta_{t}^{s}+\sum_{i \in N_{s}} \sum_{j \in N} \sum_{t \in T} Q_{i j t} \beta_{i t}^{f}+\sum_{(i, j) \in A_{c}} \sum_{u \in U} \sum_{t \in T} u C S_{i j u t} \beta_{t}^{c} \\
& +\sum_{\substack{(i, j) \in A_{p} \\
i<j}} \sum_{t \in T} \phi_{i j} V_{i j t} \beta_{t}^{p}+\sum_{\substack{(i, j) \in A_{p} \\
i<j}} \sum_{t=0}^{|T|-1} \phi_{i j}\left(V_{i j t+1}-V_{i j t}\right) \alpha_{t+1}^{p} \\
& +\sum_{(i, j) \in A_{c}} \sum_{u \in U} \sum_{t=0}^{|T|-1} u\left(C S_{i j u t+1}-C S_{i j u t}\right) \alpha_{t+1}^{c}
\end{aligned}
$$

subject to

$$
\begin{array}{ll}
P_{i t}=75 & \forall i \in N_{s}, t \in T \\
B_{i j t} \leq M Q_{i j t} & \forall(i, j) \in E, t \in T \\
Q_{i j t} \leq M B_{i j t} & \forall(i, j) \in E, t \in T \\
B_{i j t}+B_{j i t} \leq 1 & \forall(i, j) \in E, i<j, t \in T \\
P_{j t}-P_{i t} \leq M\left(1-B_{i j t}\right) & \forall(i, j) \in A_{p}, t \in T
\end{array}
$$




$$
\begin{aligned}
& \sum_{(i, j) \in E} Q_{j i t}-\sum_{(i, j) \in E} Q_{i j t}=q_{i t} \quad \forall i \in N_{d}, t \in T \\
& \sum_{(i, j) \in E} Q_{j i t}-\sum_{(i, j) \in E} Q_{i j t}=0 \quad \forall i \in N_{t} \cup N_{c}, t \in T \\
& \sum_{(i, j) \in E} Q_{i j t} \geq 0 \quad \forall i \in N_{s}, t \in T \\
& \left(P_{i t}^{2}-P_{j t}^{2}\right)-\delta_{i j} Q_{i j t}^{2} \geq M\left(B_{i j t}-1\right) \quad \forall(i, j) \in A_{p}, t \in T \\
& \left(P_{i t}^{2}-P_{j t}^{2}\right)-\delta_{i j} Q_{i j t}^{2} \leq M\left(1-B_{i j t}\right) \quad \forall(i, j) \in A_{p}, t \in T \\
& B_{i j t}+B_{j i t} \leq V_{i j t} \quad \forall(i, j) \in A_{p}, t \in T, i \leq j \\
& V_{i j t} \leq V_{i j y} \\
& \forall(i, j) \in A_{p}, i \leq j, t \in T \\
& y \in T, t \leq y \\
& 45-P_{i t} \leq M G_{i t} \quad \forall i \in N, t \in T \\
& B_{i j t}+G_{i t}-1 \leq \sum_{u \in U} C S_{i j u t} \quad \forall(i, j) \in A_{c}, t \in T \\
& \sum_{u \in U} C S_{i j u t} \leq 1 \quad \forall(i, j) \in A_{c}, t \in T \\
& B_{i j t}-\sum_{u \in U} C S_{i j u t} \geq 0 \quad \forall(i, j) \in A_{c}, t \in T \\
& \sum_{u \in U} C S_{i j u t}+\sum_{u \in U} C S_{j i u t} \leq 1 \quad \forall(i, j) \in A_{c}, i<j, t \in T \\
& C S_{i j u t} \leq C S_{i j u y} \quad \forall(i, j) \in A_{c}, i<j, t \in T, \\
& y \in T, t \leq y, u \in U \\
& P_{j t}-\left(75 \sum_{u \in U} C S_{i j u t}\right)-P_{j t}\left(1-\sum_{u \in U} C S_{i j u t}\right) \geq M\left(B_{i j t}-1\right) \quad \forall(i, j) \in A_{c}, t \in T
\end{aligned}
$$




$$
\begin{array}{ll}
P_{j t}-\left(75 \sum_{u \in U} C S_{i j u t}\right)-P_{j t}\left(1-\sum_{u \in U} C S_{i j u t}\right) \leq M\left(1-B_{i j t}\right) & \forall(i, j) \in A_{c}, t \in T \\
\sigma \sum_{u \in U} u C S_{i j u t}-\sum_{(k, i) \in A_{p} \cup A_{r}} Q_{k i t} \geq M\left(\left(\sum_{u \in U} C S_{i j u t}\right)-1\right) & \forall(i, j) \in A_{c}, t \in T \\
C S_{i j u 0}=0 & \forall(i, j) \in A_{c}, u \in U \\
V_{i j 0}=0 & \forall(i, j) \in A_{p} \\
P_{i t}, Q_{i j t} \geq 0 & \forall(i, j) \in E, t \in T \\
B_{i j t}, C S_{i j t}, V_{i j t}, G_{i t} \in\{0,1\} & \forall(i, j) \in E, t \in T
\end{array}
$$

The first term in the objective function represents the total transportation cost. The second term is associated with the total purchase cost. The third and the fourth terms represent the operating costs of compressor stations and pipelines, respectively. The fifth and the sixth terms represent the investment costs of compressor stations and a pipelines, respectively. The objective function minimizes the total cost over all periods.

Constraint (3.16) sets up the pressure level at each supply node. If there is a flow from $i$ to $j$, then constraints (3.17) and (3.18) assures that $B_{i j t}$ is equal to 1. Constraint (3.19) assures gas flows only in one direction. Constraint (3.20) determines the direction of gas flow. Constraints (3.21) and (3.22) set up the balance between incoming and outgoing flow, to and from demand and transshipment nodes, respectively. Constraint (3.23) defines that the total flow outgoing from a supply node must be greater than or equal to zero. 
Constraints (3.24) and (3.25) computes pressure drop between two nodes of pipe arcs. If there is a flow between two nodes, then constraint (3.26) assures that there is a pipe connected from one to another. Constraint (3.27) guarantees that once a pipeline is installed, it is used during planning horizon. Constraint (3.28) forces the binary variable $G$ to be equal to 1 if pressure at node $i$ is less than 45; otherwise it is set to 0. Constraint (3.29) represents the condition for installing a compressor station. If there is a flow on a compressor station arc and the pressure at inlet node is less than 45 , then a compressor station should be installed on that arc. Constraint (3.30) shows that only one type of compressor stations can be built on an arc. Constraint (3.31) assures that if there is not any flow between two nodes of a compressor station arc, then the arc should not be used. Constraint (3.32) shows that a compressor station can be installed only in one direction. Constraint (3.33) assures that once a compressor station is installed, it is used during planning horizon. Constraints (3.34) and (3.35) set up the pressure to 75 bar at outlet nodes of compressor stations arcs, if they are installed; otherwise, pressure at inlet and outlet nodes should be equal. Constraint (3.36) represents that the flow rate through a compressor station arc should be less than the total capacity of a compressor station. Constraint (3.37) sets the existence of a compressor station in period 0 to 0 . Constraint (3.38) sets the existence of a pipeline in period 0 to 0 . Constraints (3.39) and (3.40) represent the ranges of the variables. 


\section{THE SOLUTION METHOD AND COMPUTATIONAL STUDY}

\subsection{The Solution Method}

This study proposes a mathematical optimization model that handles many pipeline gas transmission network problems. The problem was modeled as a MINLP model with AMPL (A Modeling Language for Mathematical Programming) [12]. The computability of the model was assessed by using Bonmin [4] which implements $\mathrm{BB}$ algorithm to solve MINLP models with non-convex functions.

The mathematical optimization method can be used to find feasible solutions as long as the mathematical model is a good representative of the problem. An optimization method usually uses simplified models, but, it may find optimum solutions for certain objectives, subject to the number of constraints that have been defined before.

Mathematical methods can be classified as local or global search methods. The global solution method is used to find a global optimum by reducing the gap between the lower and upper bounds of the problem. The local solution method aims to achieve a local optimum by generating new solutions using a neighborhood search.

A number of solution techniques, including combinatorial and nonlinear optimization, have been used in many fields of the mathematical programming. These methods can be classified as exact (analytical) and approximate (numerical solutions). The exact solution method is bounded for specific problems. For large real-world problems, this method may be time consuming. Approximate solutions express the system in numerical functions. Numerical methods look for solutions within reasonable computational times by solving equations for known parameters and variables. 
A mathematical model represents a real-world problem by the objective function and constraints (if any). The search space is the set of all feasible solutions that are bounded by constraints. Combinatorial optimization proposes efficient solution methods in order to handle problems with large feasible regions [5].

In NG pipeline transportation optimization problems, a feasible region is defined as non-convex. The size and complexity of the problem increase and the feasible region expands when, for example, new demand nodes are considered, so new pipelines and compressor stations are required in the system. Thus, finding the optimal design of the network that minimizes the total cost requires the theory and the application of nonlinearly constrained optimization.

\subsubsection{Mixed Integer Nonlinear Programming}

MINLP is a mathematical programming approach with nonlinear functions in the objective function and constraints. MINLP has been used in many applications from various areas, including chemical sciences, logistics, engineering design, manufacturing, energy generation and distribution. A general MINLP can be formulated as

$$
\operatorname{Min} f(x)
$$

subject to

$$
\begin{array}{ll}
g_{j}(x) \leq 0 & \forall j=1,2, \ldots, m \\
l_{i} \leq x_{i} \leq u_{i} & \forall i=1,2, \ldots, n \\
x \in \mathbb{Z}^{r} \mathbf{x} \mathbb{R}^{n-r} &
\end{array}
$$

where $f: \mathbb{R}^{n} \rightarrow \mathbb{R}$ and $g_{j}: \mathbb{R}^{n} \rightarrow \mathbb{R}, \forall \mathrm{j}=1,2, \ldots, \mathrm{m}$, are generally non-convex functions; $n$ is the number of variables, $r$ is the number of integer variables and $x$ is 
the n-vector of variables where $l_{i}$ and $u_{i}$ determine lower and upper bounds on the variables [3].

MINLP problems are very difficult to solve, because they include the difficulties of both MIP and nonlinear programming (NLP), which are MINLP's subclasses. As MIP has a combinatorial nature, non-convex and convex NLP problems are hard to solve. Since both MIP and NLP are NP-complete problems, solving MINLP can be challenging.

Non-convex MINLPs are typically harder to solve optimally than convex problems. For convex MINLPs, an initial lower bound can be computed by solving the continuous relaxation of the problem. This relaxation will be a convex NLP so that it is relatively easy to solve. On the other hand, a continuous relaxation of a non-convex MINLP is a non-convex NLP, which is classified as a NP-hard problem. [3]

Defining a NG transmission network requires using nonlinear equalities and binary variables. Therefore, MINLP is used to describe the problem. Because of the characteristics of the pressure and flow rate relation, the feasible region of the problem is non-convex. Typically, the BB algorithm is used to solve nonlinear non-convex problems.

\subsubsection{The Branch-and-Bound Algorithm}

Bonmin solves non-convex MINLP problems using BB algorithm. This algorithm is the method for global optimization in non-convex problems [17]. The method is to branch on all variables before closing the gap between the lower and upper bound on the globally optimal objective value. These algorithms can be slow. In some cases computational times increase exponentially with problem size [7].

The space of all feasible solutions (the search space) is repeatedly partitioned into submodels. After tightening the bounds on the discrete variables to integer 
values, non-integer solutions are eliminated. A tightened submodel is solved by using the optimal solution to the previous looser submodel. In the case of minimization, the objective function values of submodels are assumed to be lower bounds in the restricted search space. The search continues to examine further nodes in the tree until a feasible solution with an objective function value that is no greater than the bound for any submodel [27].

For constrained optimization problems with discrete variables and/or non-convex objective function or constraints, exact solution methods are inefficient. BB methods are one of the best ways to obtain globally optimal solutions to nonlinear programming problems with non-convex functions [17].

\subsubsection{Overview of AMPL and Bonmin}

Modeling language systems are widely used tools in the development of mathematical models. One of the most widely used modeling languages is AMPL [12]. AMPL is an algebraic modeling language for formulating and solving high-complexity problems for a large-scale mathematical computation. Linear and nonlinear optimization problems with discrete or continuous variables can be modeled by AMPL. AMPL's syntax is similar to mathematical notations of optimization problems. This allows for a comprehensible definition of models. In this study, AMPL is used to describe the problem model.

AMPL solver options comprise a considerable number of optimization tools including, CPLEX[16], Gurobi[14], MINOS[21], and KNITRO[7] . The solvers differ from each other in such a way that each apply different methods to solve problems with a given proven optimality and the characteristics of the models they handle.

After modeling with AMPL, the problem will be solved by Bonmin (Basic Open-

source Nonlinear Mixed Integer Programming) [4]. The aim is to assess the com- 
putability of the model. Bonmin is an online solver of the Computational Infrastructure for Operations Research (COIN-OR) [18], which is an initiative project to encourage the development of open-source software for the operations research community. Bonmin and many other COIN-OR solvers can be accessed on-line through the NEOS Server [10]. Optimization problems are solved by solvers automatically with minimal input from the user. Users upload the problem's formulation to the server as an input. AMPL, GAMS[25] or MATLAB[19] can be used to define problems. All other information required by the optimization solver is determined automatically. For each problem type, there are different optimization solvers. For instance, BARON[26], Bonmin or Couenne[2] can be used to solve MINLP.

Bonmin is an open source code for solving general MINLP problems in AMPL , GAMS and $\mathrm{C} / \mathrm{C}++$ format. The methods that Bonmin uses exact algorithms when nonlinear functions are convex. Bonmin implements four different algorithms for solving MINLPs: (1) a NLP-based BB algorithm, (2) an outer-approximation based BB algorithm, (3) an outer-approximation based decomposition algorithm, and (4) a hybrid outer-approximation/NLP based branch-and-cut algorithm. A NLP-based BB algorithm solves a continuous nonlinear program at each node of the search tree and branches on variables [4].

\subsection{Computational Study and Analysis}

In this section, an evaluation of the computability of the proposed model is provided. The network problems are generated by using the assumptions and the parameters that were discussed previously. Different variations of the model are tested. Outputs of the problems are compared, and the robustness of solutions is discussed.

Input data are realistic data, i.e., demand quantities are randomly generated according to the current usage in Turkey by using uniform distribution. Then, using 
these data, monthly, seasonal and yearly data were computed. Monthly demands vary in each period according to the season. For instance, peak demand occurs in the winter session between October and March. Summer session is between April and September. This season holds the lowest demand in a year. Seasonal demands are the summation of demands in a season while yearly demand is the total of the two seasons' demand values. Typically, demand increases over 5 years between $5 \%$ and $15 \%$. In this study, it is assumed that demand increases by $1 \%$ in each year. The structure of the studied networks is inspired by the transmission network of Turkey. The position of nodes is consistent with their geographical location in the real network.

Flow directions are designated by the constraints and no flow direction is assigned a priori. The length and diameter of pipeline arcs are established before running the test instances. The pressure of gas at supply nodes is fixed to 75 bar. The pressure at other nodes is limited by the maximum allowable operating pressure of the pipeline. The operating pressures of the pipelines are not given as problem data, but they are computed during the optimization run by satisfying constraints.

As a result of numerical experiments, the model is expected to have a treestructured network that there is at least one path to any demand node and at least one path from any supply node. It is also anticipated that all demand and capacity requirements are satisfied in all periods, and pressures at nodes are in the allowed range. Once a pipeline or a compressor station is installed, the model will adjust gas flow accordingly in latter periods.

The following data has been decided to be used due to the evidence suggested by many test instances. Length and resistance of each pipe type are given in Table 4.1 and demand data are shown in Table 4.2. 
Table 4.1: Monthly Demand Data (mmscm)

\begin{tabular}{|c|c|c|c|c|c|c|c|c|c|c|c|c|}
\hline \multirow{2}{*}{$\begin{array}{c}\text { Demand } \\
\text { Nodes }\end{array}$} & \multicolumn{12}{|c|}{ Periods } \\
\hline & JAN & FEB & MAR & APR & MAY & JUN & JUL & AUG & SEP & OCT & NOV & DEC \\
\hline 2 & 158.98 & 144.53 & 98.71 & 81.58 & 74.16 & 67.42 & 61.29 & 55.72 & 89.74 & 108.58 & 119.44 & 131.39 \\
\hline 3 & 163.28 & 148.43 & 101.38 & 83.79 & 76.17 & 69.25 & 62.95 & 57.23 & 92.17 & 111.52 & 122.67 & 134.94 \\
\hline 5 & 139.83 & 127.12 & 86.82 & 71.75 & 65.23 & 59.30 & 53.91 & 49.01 & 78.93 & 95.50 & 105.05 & 115.56 \\
\hline 6 & 141.37 & 128.52 & 87.78 & 72.55 & 65.95 & 59.96 & 54.50 & 49.55 & 79.80 & 96.56 & 106.21 & 116.84 \\
\hline 8 & 114.59 & 104.17 & 71.15 & 58.80 & 53.46 & 48.60 & 44.18 & 40.16 & 64.68 & 78.27 & 86.09 & 94.70 \\
\hline 9 & 116.03 & 105.48 & 72.04 & 59.54 & 54.13 & 49.21 & 44.73 & 40.67 & 65.50 & 79.25 & 87.17 & 95.89 \\
\hline 10 & 176.96 & 160.87 & 109.88 & 90.81 & 82.55 & 75.05 & 68.23 & 62.02 & 99.89 & 120.87 & 132.95 & 146.25 \\
\hline 12 & 87.58 & 79.62 & 54.38 & 44.94 & 40.86 & 37.14 & 33.77 & 30.70 & 49.44 & 59.82 & 65.80 & 72.38 \\
\hline 17 & 115.34 & 104.86 & 71.62 & 59.19 & 53.81 & 48.92 & 44.47 & 40.43 & 65.11 & 78.78 & 86.66 & 95.32 \\
\hline 18 & 176.88 & 160.80 & 109.83 & 90.77 & 82.51 & 75.01 & 68.19 & 61.99 & 99.84 & 120.81 & 132.89 & 146.18 \\
\hline 19 & 132.47 & 120.43 & 82.25 & 67.98 & 61.80 & 56.18 & 51.07 & 46.43 & 74.78 & 90.48 & 99.53 & 109.48 \\
\hline 20 & 147.66 & 134.23 & 91.68 & 75.77 & 68.88 & 62.62 & 56.93 & 51.75 & 83.35 & 100.85 & 110.94 & 122.03 \\
\hline 22 & 158.24 & 143.86 & 98.26 & 81.20 & 73.82 & 67.11 & 61.01 & 55.46 & 89.32 & 108.08 & 118.89 & 130.78 \\
\hline 25 & 84.91 & 77.19 & 52.72 & 43.57 & 39.61 & 36.01 & 32.74 & 29.76 & 47.93 & 57.99 & 63.79 & 70.17 \\
\hline 26 & 176.89 & 160.81 & 109.83 & 90.77 & 82.52 & 75.02 & 68.20 & 62.00 & 99.85 & 120.82 & 132.90 & 146.19 \\
\hline 30 & 113.34 & 103.03 & 70.37 & 58.16 & 52.87 & 48.07 & 43.70 & 39.72 & 63.98 & 77.41 & 85.15 & 93.67 \\
\hline 31 & 131.88 & 119.89 & 81.89 & 67.67 & 61.52 & 55.93 & 50.84 & 46.22 & 74.44 & 90.07 & 99.08 & 108.99 \\
\hline 33 & 160.04 & 145.49 & 99.37 & 82.13 & 74.66 & 67.87 & 61.70 & 56.09 & 90.34 & 109.31 & 120.24 & 132.26 \\
\hline 35 & 71.84 & 65.31 & 44.61 & 36.86 & 33.51 & 30.47 & 27.70 & 25.18 & 40.55 & 49.07 & 53.97 & 59.37 \\
\hline 37 & 71.39 & 64.90 & 44.33 & 36.64 & 33.31 & 30.28 & 27.53 & 25.02 & 40.30 & 48.76 & 53.64 & 59.00 \\
\hline 39 & 63.28 & 57.52 & 39.29 & 32.47 & 29.52 & 26.83 & 24.40 & 22.18 & 35.72 & 43.22 & 47.54 & 52.29 \\
\hline 40 & 71.25 & 64.77 & 44.24 & 36.56 & 33.24 & 30.22 & 27.47 & 24.97 & 40.22 & 48.67 & 53.53 & 58.89 \\
\hline 42 & 109.69 & 99.72 & 68.11 & 56.29 & 51.17 & 46.52 & 42.29 & 38.44 & 61.92 & 74.92 & 82.41 & 90.65 \\
\hline 44 & 180.11 & 163.74 & 111.84 & 92.43 & 84.02 & 76.39 & 69.44 & 63.13 & 101.67 & 123.02 & 135.32 & 148.85 \\
\hline 46 & 135.71 & 123.37 & 84.27 & 69.64 & 63.31 & 57.56 & 52.32 & 47.57 & 76.61 & 92.69 & 101.96 & 112.16 \\
\hline 48 & 114.45 & 104.05 & 71.06 & 58.73 & 53.39 & 48.54 & 44.13 & 40.11 & 64.60 & 78.17 & 85.99 & 94.59 \\
\hline 49 & 132.29 & 120.26 & 82.14 & 67.88 & 61.71 & 56.10 & 51.00 & 46.37 & 74.67 & 90.35 & 99.39 & 109.33 \\
\hline 50 & 186.19 & 169.27 & 115.61 & 95.55 & 86.86 & 78.96 & 71.79 & 65.26 & 105.10 & 127.17 & 139.89 & 153.88 \\
\hline 52 & 129.62 & 117.83 & 80.48 & 66.51 & 60.47 & 54.97 & 49.97 & 45.43 & 73.17 & 88.53 & 97.38 & 107.12 \\
\hline 55 & 53.42 & 48.56 & 33.17 & 27.41 & 24.92 & 22.65 & 20.59 & 18.72 & 30.15 & 36.48 & 40.13 & 44.15 \\
\hline 58 & 186.71 & 169.74 & 115.93 & 95.81 & 87.10 & 79.18 & 71.98 & 65.44 & 105.39 & 127.53 & 140.28 & 154.31 \\
\hline 59 & 121.83 & 110.75 & 75.65 & 62.52 & 56.83 & 51.67 & 46.97 & 42.70 & 68.77 & 83.21 & 91.53 & 100.69 \\
\hline 60 & 70.11 & 63.73 & 43.53 & 35.98 & 32.71 & 29.73 & 27.03 & 24.57 & 39.57 & 47.88 & 52.67 & 57.94 \\
\hline 62 & 172.63 & 156.93 & 107.19 & 88.58 & 80.53 & 73.21 & 66.55 & 60.50 & 97.44 & 117.91 & 129.70 & 142.67 \\
\hline 63 & 175.19 & 159.26 & 108.78 & 89.90 & 81.73 & 74.30 & 67.54 & 61.40 & 98.89 & 119.66 & 131.62 & 144.78 \\
\hline 66 & 135.11 & 122.83 & 83.89 & 69.33 & 63.03 & 57.30 & 52.09 & 47.36 & 76.27 & 92.28 & 101.51 & 111.66 \\
\hline 68 & 77.57 & 70.52 & 48.16 & 39.80 & 36.19 & 32.90 & 29.91 & 27.19 & 43.78 & 52.98 & 58.28 & 64.10 \\
\hline 69 & 177.22 & 161.11 & 110.04 & 90.94 & 82.67 & 75.16 & 68.32 & 62.11 & 100.03 & 121.04 & 133.14 & 146.46 \\
\hline 71 & 76.51 & 69.55 & 47.50 & 39.26 & 35.69 & 32.45 & 29.50 & 26.82 & 43.19 & 52.26 & 57.48 & 63.23 \\
\hline 73 & 93.12 & 84.66 & 57.82 & 47.79 & 43.44 & 39.49 & 35.90 & 32.64 & 52.56 & 63.60 & 69.96 & 76.96 \\
\hline 79 & 62.65 & 56.96 & 38.90 & 32.15 & 29.23 & 26.57 & 24.16 & 21.96 & 35.37 & 42.79 & 47.07 & 51.78 \\
\hline 81 & 76.31 & 69.37 & 47.38 & 39.16 & 35.60 & 32.36 & 29.42 & 26.74 & 43.07 & 52.12 & 57.33 & 63.06 \\
\hline 82 & 71.96 & 65.42 & 44.68 & 36.93 & 33.57 & 30.52 & 27.74 & 25.22 & 40.62 & 49.15 & 54.07 & 59.47 \\
\hline 83 & 160.80 & 146.19 & 99.85 & 82.52 & 75.02 & 68.20 & 62.00 & 56.36 & 90.77 & 109.83 & 120.81 & 132.90 \\
\hline 85 & 43.71 & 39.74 & 27.14 & 22.43 & 20.39 & 18.54 & 16.85 & 15.32 & 24.68 & 29.86 & 32.84 & 36.13 \\
\hline 87 & 53.32 & 48.47 & 33.11 & 27.36 & 24.87 & 22.61 & 20.56 & 18.69 & 30.10 & 36.42 & 40.06 & 44.07 \\
\hline 88 & 155.78 & 141.62 & 96.73 & 79.94 & 72.67 & 66.07 & 60.06 & 54.60 & 87.93 & 106.40 & 117.04 & 128.74 \\
\hline 90 & 112.56 & 102.33 & 69.89 & 57.76 & 52.51 & 47.74 & 43.40 & 39.45 & 63.54 & 76.88 & 84.57 & 93.03 \\
\hline
\end{tabular}


Table 4.2: Pipeline Arc Lengths and Resistances

\begin{tabular}{|c|c|c|c|c|c|c|c|c|}
\hline Arc & $\phi_{i j} \quad(\mathbf{k m})$ & $\delta_{i j}$ & Arc & $\phi_{i j} \quad(\mathrm{~km})$ & $\delta_{i j}$ & Arc & $\phi_{i j} \quad(\mathrm{~km})$ & $\delta_{i j}$ \\
\hline 1,2 & 25 & 1.344 & 38,40 & 5 & 0.269 & 72,73 & 10 & 0.537 \\
\hline 2,3 & 25 & 1.344 & 38,39 & 20 & 1.075 & 72,74 & 100 & 5.374 \\
\hline 3,4 & 25 & 1.344 & 34,41 & 50 & 2.687 & 74,75 & 50 & 2.687 \\
\hline 4,5 & 30 & 1.612 & 41,42 & 45 & 2.418 & 75,76 & 10 & 0.537 \\
\hline 5,6 & 30 & 1.612 & 43,44 & 5 & 0.269 & 75,85 & 50 & 2.687 \\
\hline 6,7 & 30 & 1.612 & 43,45 & 50 & 2.687 & 85,86 & 5 & 0.269 \\
\hline 7,9 & 60 & 3.225 & 45,46 & 45 & 2.418 & 86,87 & 10 & 0.537 \\
\hline 7,8 & 30 & 1.612 & 45,47 & 50 & 2.687 & 86,88 & 5 & 0.269 \\
\hline 9,10 & 60 & 3.225 & 47,48 & 75 & 4.031 & 77,78 & 10 & 0.537 \\
\hline 4,11 & 50 & 2.687 & 48,49 & 75 & 4.031 & 78,79 & 5 & 0.269 \\
\hline 11,12 & 5 & 0.269 & 47,50 & 50 & 2.687 & 78,80 & 25 & 1.344 \\
\hline 11,13 & 50 & 2.687 & 29,51 & 100 & 5.374 & 80,81 & 20 & 1.075 \\
\hline 14,15 & 50 & 2.687 & 51,52 & 5 & 0.269 & 80,82 & 20 & 1.075 \\
\hline 15,16 & 40 & 2.150 & 51,53 & 50 & 2.687 & 80,83 & 25 & 1.344 \\
\hline 16,19 & 20 & 1.075 & 53,54 & 25 & 1.344 & 83,84 & 50 & 2.687 \\
\hline 16,17 & 20 & 1.075 & 54,55 & 5 & 0.269 & 89,90 & 10 & 0.537 \\
\hline 16,18 & 20 & 1.075 & 54,56 & 25 & 1.344 & 74,89 & 10 & 0.537 \\
\hline 19,20 & 20 & 1.075 & 56,57 & 20 & 1.075 & 41,91 & 25 & 1.344 \\
\hline 15,21 & 50 & 2.687 & 57,58 & 20 & 1.075 & 43,92 & 25 & 1.344 \\
\hline 21,22 & 5 & 0.269 & 57,59 & 20 & 1.075 & 31,93 & 25 & 1.344 \\
\hline 21,23 & 50 & 2.687 & 56,60 & 50 & 2.687 & 32,94 & 25 & 1.344 \\
\hline 23,24 & 50 & 2.687 & 50,61 & 50 & 2.687 & 18,20 & 20 & 1.075 \\
\hline 24,25 & 10 & 0.537 & 60,62 & 60 & 3.225 & 25,26 & 20 & 1.075 \\
\hline 24,26 & 10 & 0.537 & 60,63 & 60 & 3.225 & 33,39 & 20 & 1.075 \\
\hline 23,27 & 50 & 2.687 & 60,96 & 50 & 2.687 & 37,42 & 20 & 1.075 \\
\hline 28,29 & 50 & 2.687 & 64,97 & 50 & 2.687 & 42,46 & 20 & 1.075 \\
\hline 29,30 & 5 & 0.269 & 64,65 & 100 & 5.374 & 55,58 & 20 & 1.075 \\
\hline 29,31 & 50 & 2.687 & 65,66 & 5 & 0.269 & 17,26 & 50 & 2.687 \\
\hline 32,34 & 50 & 2.687 & 65,67 & 75 & 4.031 & 17,18 & 30 & 1.612 \\
\hline 32,33 & 5 & 0.269 & 67,68 & 50 & 2.687 & 79,88 & 50 & 2.687 \\
\hline 34,35 & 10 & 0.537 & 68,69 & 100 & 5.374 & 79,81 & 30 & 1.612 \\
\hline 35,36 & 10 & 0.537 & 67,70 & 75 & 4.031 & 59,63 & 20 & 1.075 \\
\hline 36,38 & 100 & 5.374 & 70,71 & 5 & 0.269 & 70,95 & 50 & 2.687 \\
\hline 36,37 & 5 & 0.269 & 53,72 & 5 & 0.269 & & & \\
\hline
\end{tabular}


The investment costs of pipeline and compressor station installations will be decreasing in each year due to straight line depreciation method. For example, the investment cost of a compressor station in the first year is not the same as the investment cost in the second year. Moreover, the operating costs of pipelines decrease while compressor station operating costs increase in each year. Transportation cost also varies depending on the total costs of compressor station and pipelines. Since gas prices increase $2 \%$ each year, purchase cost changes in each period.

The numerical examples were solved in less than 8 hours with an allowable gap of $5 \%$. However, all solutions are obtained with an integer gap less than $0 \%$.

\subsubsection{Experiment 1: Various Problem Sizes}

In this experiment, the computational performance of the proposed model is examined by different problem sizes. The subjects of the experiment are three realistic different transmission networks, one with 31, the other with 66 and another with 97 nodes. Output of each test instance provides information about the design of the network, the location of pipeline and compressor station installations, the amount of gas that should be purchased at the beginning of the period.

The pipeline lengths in both networks vary from $5 \mathrm{~km}$ to $100 \mathrm{~km}$ and the diameter of each pipeline is 30 inches. The total pipeline length of the 31-node network is 1040 $\mathrm{km}$, of the 66 -node network is $2440 \mathrm{~km}$, and of the 97 -node network is $3500 \mathrm{~km}$.

31-node problem network has 32 pipe arcs, and 2 compressor arcs. The system was constituted with 2 supply, 16 demand, 9 transshipment nodes, and 2 compressor stations. Figure 4.1 shows the underlying network of the problem. The mathematical formulation of this network for one period has 181 binary, 149 continuous variables, as well as 140 nonlinear, 403 linear, 105 equality, and 438 inequality constraints. 


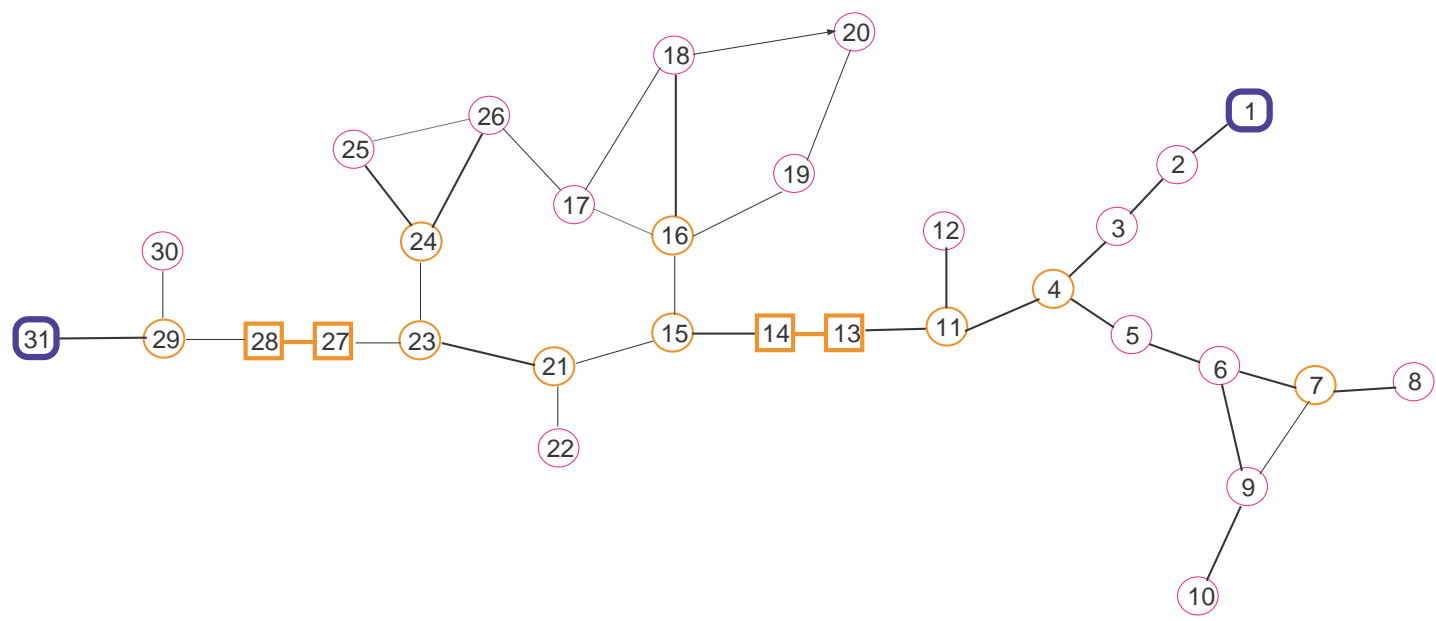

Figure 4.1: A Network with 31 Nodes

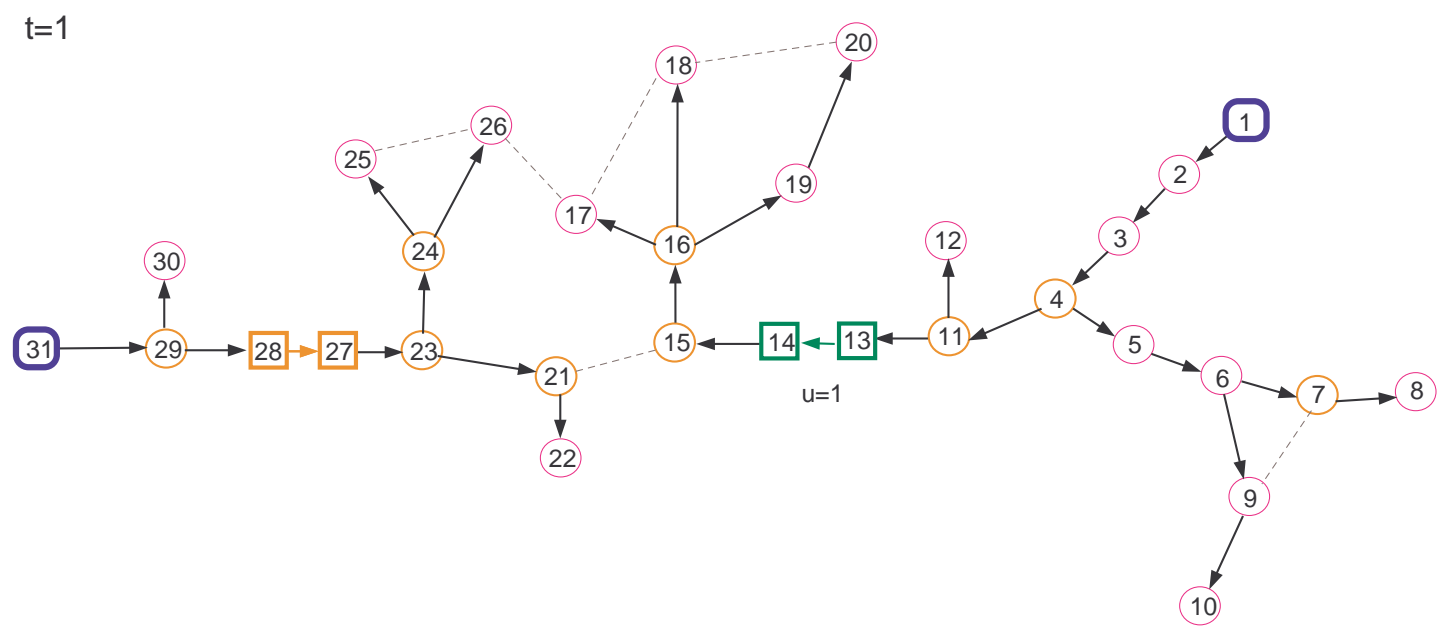

Figure 4.2: Solution to the 31-Node Network for 1-period

As shown in Figure 4.2, the output of the problem with one period consists of two tree networks, and there is at least one path to each demand node. Compressor stations at nodes 13-14 and 27-28 are installed and they will be available during 12 
years. Pressures at nodes are given in Table 4.3 and Figure 4.3 shows the daily flow rate in the period.

Table 4.3: Pressure Levels at Nodes in the 31-Node Network for 1-period

\begin{tabular}{|c|c|c|c|}
\hline Node & Pressure (bar) & Node & Pressure (bar) \\
\hline 1 & 75.00 & 17 & 71.19 \\
2 & 62.81 & 18 & 70.79 \\
3 & 50.86 & 19 & 71.12 \\
4 & 39.03 & 20 & 70.70 \\
5 & 33.74 & 21 & 72.36 \\
6 & 29.91 & 22 & 72.33 \\
7 & 29.73 & 23 & 72.59 \\
8 & 29.55 & 24 & 71.41 \\
9 & 27.49 & 25 & 71.39 \\
10 & 26.55 & 26 & 71.27 \\
11 & 33.01 & 27 & 75.00 \\
12 & 32.99 & 28 & 68.84 \\
13 & 27.93 & 29 & 71.38 \\
14 & 75.00 & 30 & 71.36 \\
15 & 72.91 & 31 & 75.00 \\
16 & 71.19 & & \\
\hline
\end{tabular}

As shown in the Table 4.3, while gas flows, pressure drops because of the resistance and flow losses. Even though the pressure at the node 28 is above 45 bar, compressor station is installed at that node in order to balance the pressure drop and flow rate. Furthermore, at some nodes pressure is below 45 bar, so system analysts can decide to locate compressor stations at other nodes considering cost and geotechnical factors.

As shown in Figure 4.3, at least 49.41 mmscm gas must be delivered from suppliers in a year; $35.36 \mathrm{mmscm}$ from supplier 1, and $14.05 \mathrm{mmscm}$ from supplier 2. 


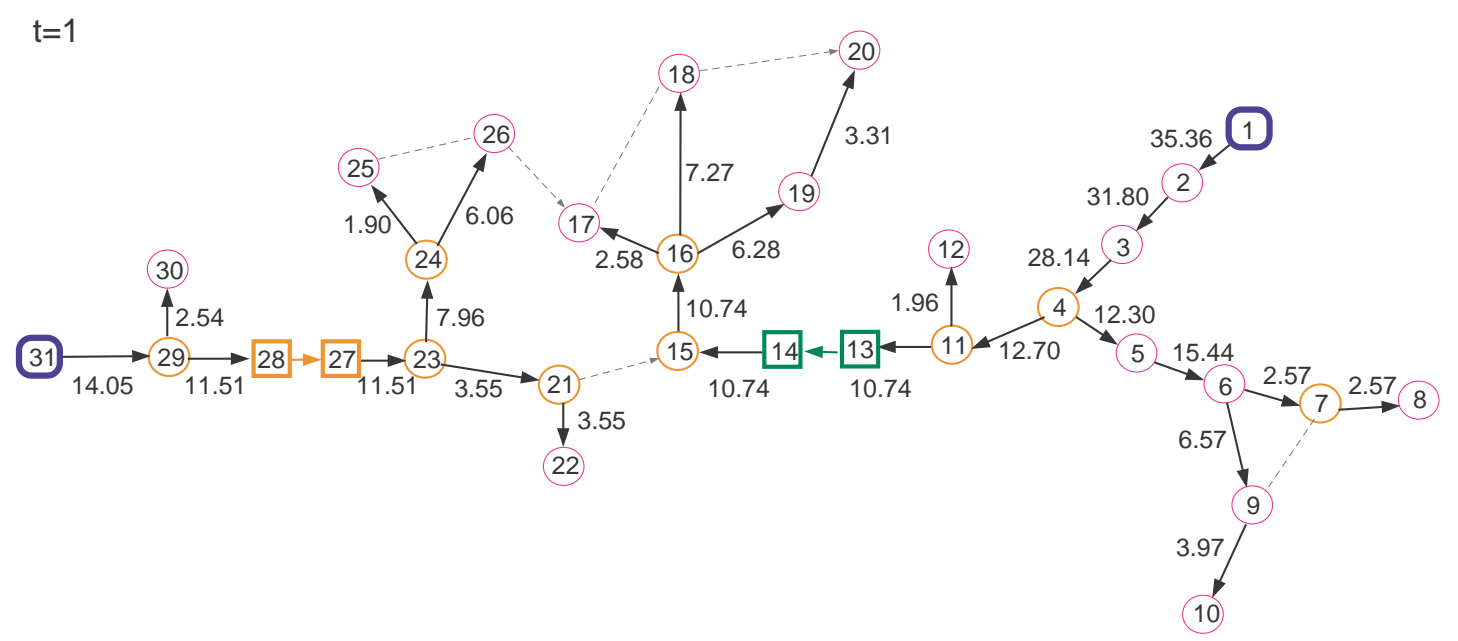

Figure 4.3: Flow Rates in the 31-Node Network for 1-period

66-node problem network has 71 pipe arcs, and 4 compressor arcs. This system was designed with 3 supply, 33 demand, 22 transshipment nodes and 4 compressor stations. Figure 4.4 shows the underlying network of one period problem. The mathematical formulation of this network for one period has 392 binary, 234 continuous variables, as well as 304 nonlinear, 869 linear, 226 equality, and 947 inequality constraints. 


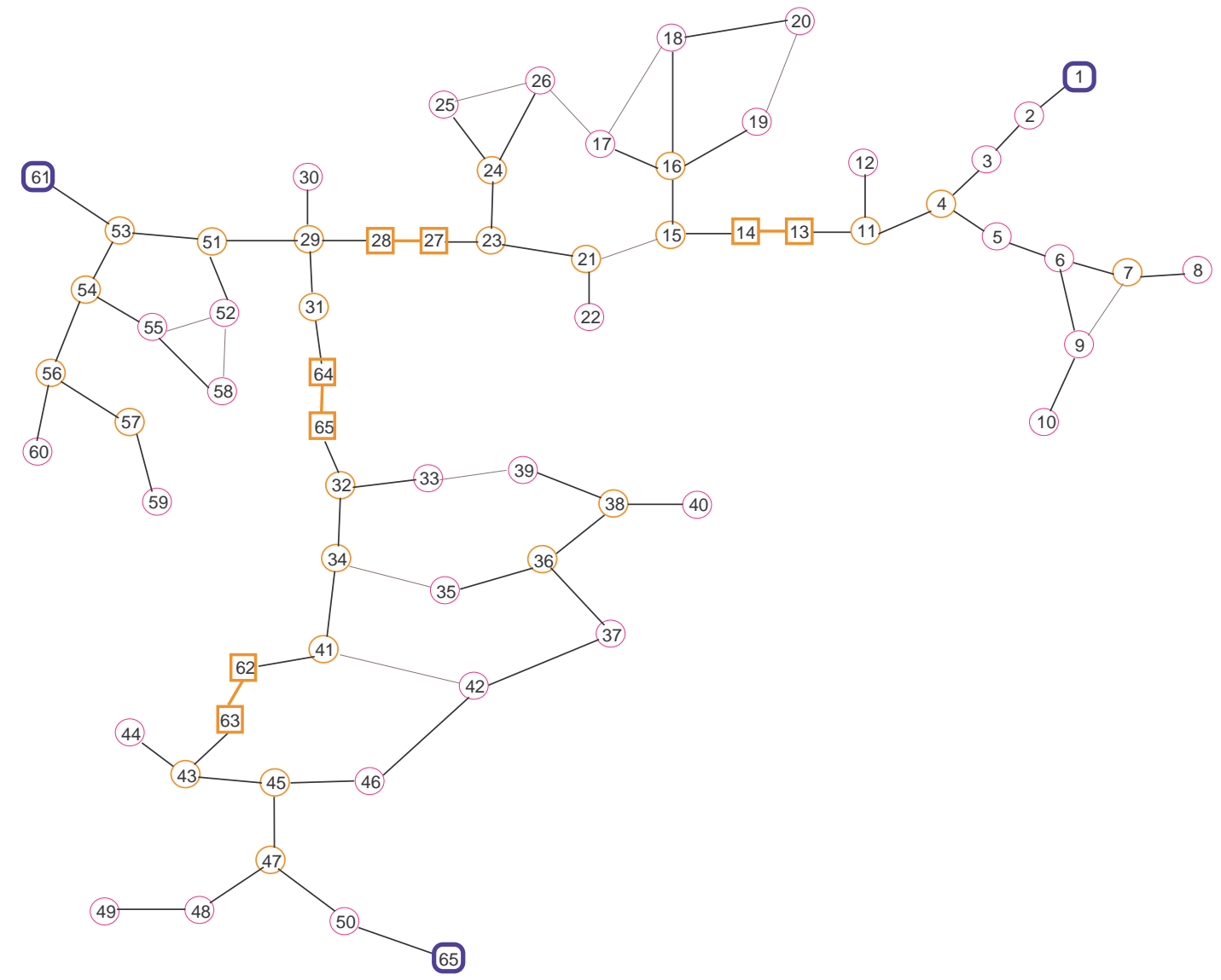

Figure 4.4: A Network with 66 Nodes 


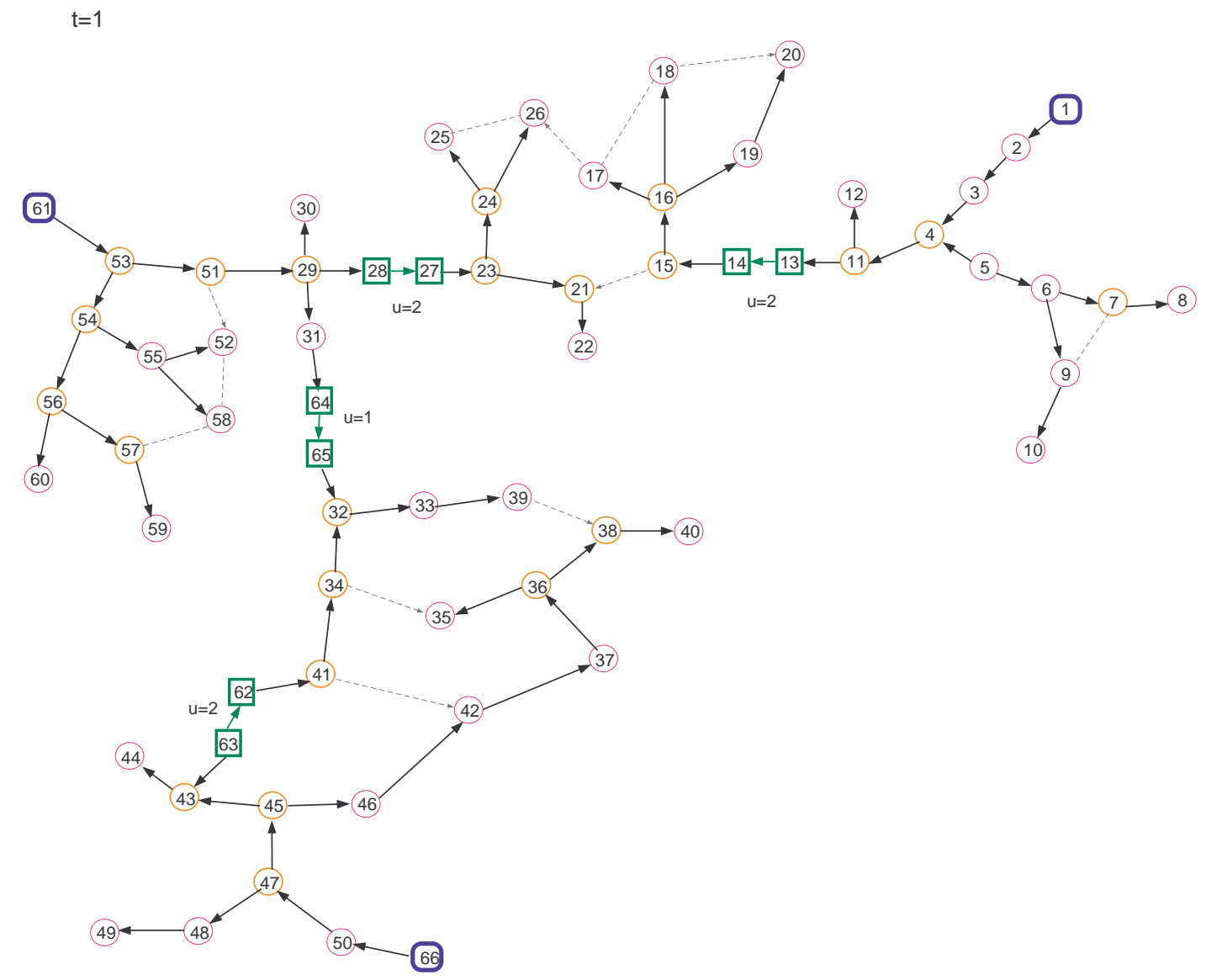

Figure 4.5: Solution to the 66-Node Network for 1-period

As shown in Figure 4.5, the problem solution consists of two tree networks, and there is at least one path to each demand node. Compressor stations are needed at each compressor station node during the period.

97-node problem network has 102 pipe arcs, and 6 compressor arcs. This system was designed with 4 supply, 48 demand, and 33 transshipment nodes. Figure 4.6 shows the underlying network of one period problem. The mathematical formulation of this network for one period has 563 binary, 340 continuous variables, as well as 436 nonlinear, 1252 linear, 328 equality, and 1360 inequality constraints. 


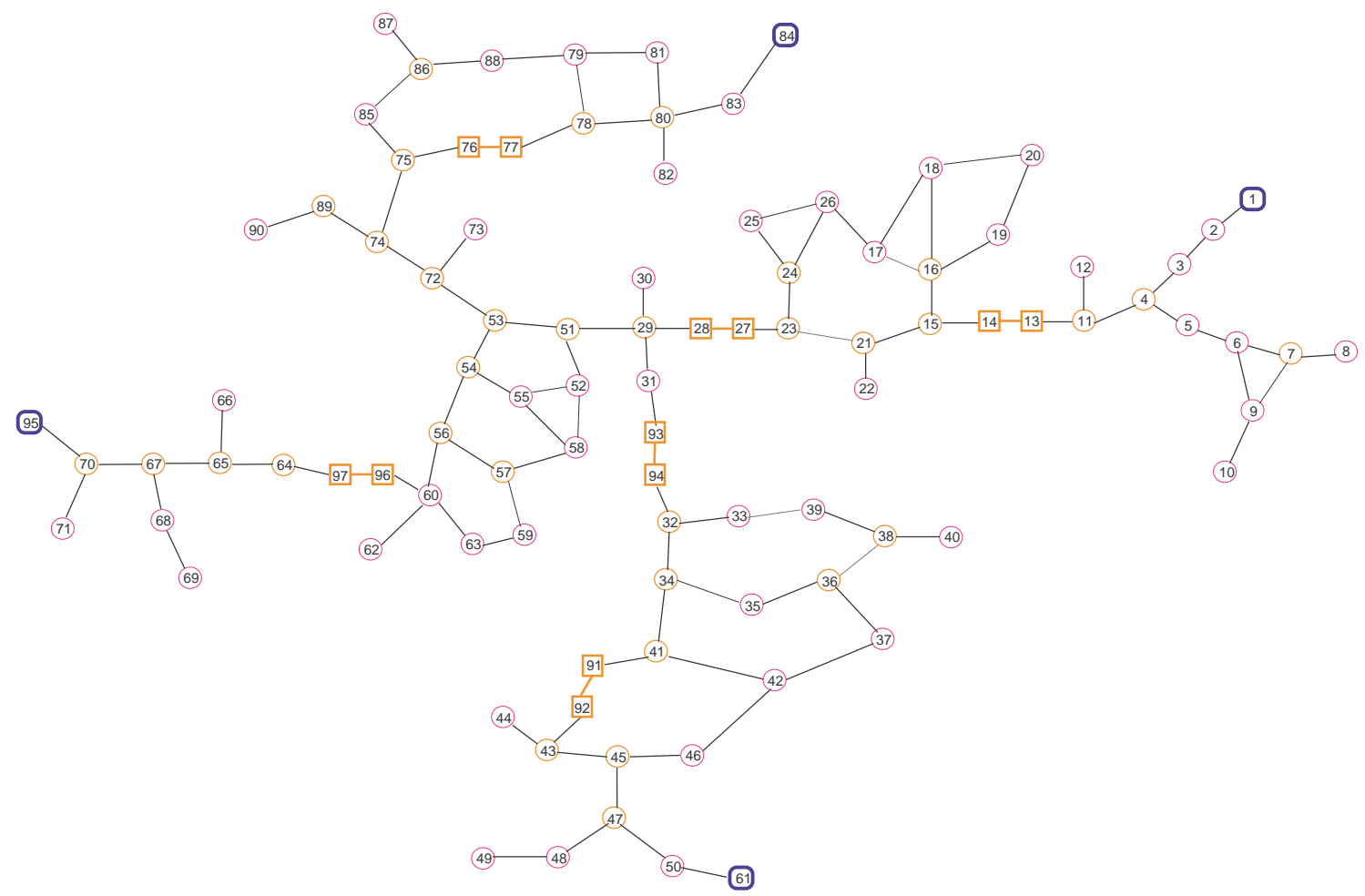

Figure 4.6: A Network with 97 Nodes 


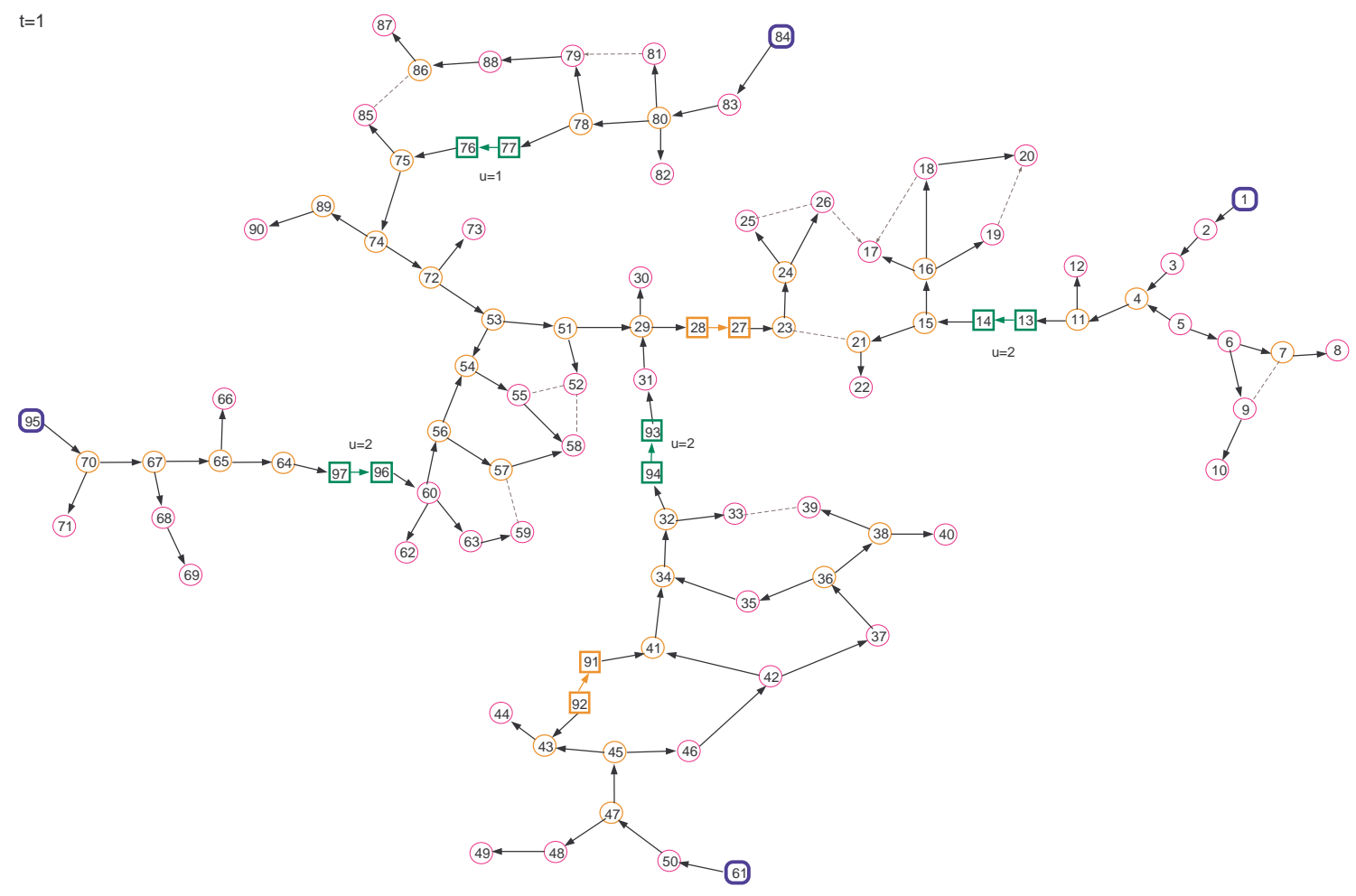

Figure 4.7: Solution to the 97-Node Network for 1-period

Figure 4.7, the problem solution consists of two tree networks, and there is at least one path to each demand node. 4 compressor stations at nodes 13-14, 76-77, 93-94, and 96-97, are installed in the system. Gas is supplied from all suppliers.

Previous test instances showed that for larger size problems the computational time exceeds 8 hours. Since they have more arcs and nodes, the model tries every arc to minimize the total flow and cost. Therefore, the 66-node and 97-node problems are solved for 1-period.

The optimal total cost and the solution time (CPU time) of each problem instance are given in Table 4.4 . 
Table 4.4: Optimal Costs and Solution Times for Different Sized Networks

\begin{tabular}{|c|r|r|r|}
\hline Problem & Best objective value $(\$)$ & CPU time (sec) & Gap \% \\
\hline 31-node & $82,140,847,000$ & 47.37 & 0.0013 \\
66-node & $163,811,060,000$ & 258.41 & 0.00276 \\
97-node & $339,252,770,000$ & 709.80 & 0.016 \\
\hline
\end{tabular}

As presented in the Table 4.4, the optimal solution to the single period with various network sizes can be obtained in less than one hour. However, the computational time increases with the number of nodes and arcs. Therefore, for the large size problems, the whole network can be divided into small networks and each network can be solved individually.

\subsubsection{Experiment 2: The Effect of the Period Lengths}

In this experiment, the model performance with different number of periods is examined. The aim of this test study is to find the optimum planning horizon length for the NG transmission network with varying levels of demand. The optimal planning horizon not the only gives the minimum cost but also requires fewer changes over 12 year span. System analysts try to operate the existing network structure with minimal changes over long periods, because installing new pipelines/compressor stations, and not using the existing ones, may cause considerable expenses.

Typically, demand projections, and compressor station and pipeline installation decisions are made for 10 years. In this study, a 12 year span is chosen as a long term planning horizon considering solution times of problems. It is also an appropriate time period to observe changes on the network structure. Thus, various instances based on the 31-node network are generated for different number of periods, but with the same planning horizon length of 12 years. 


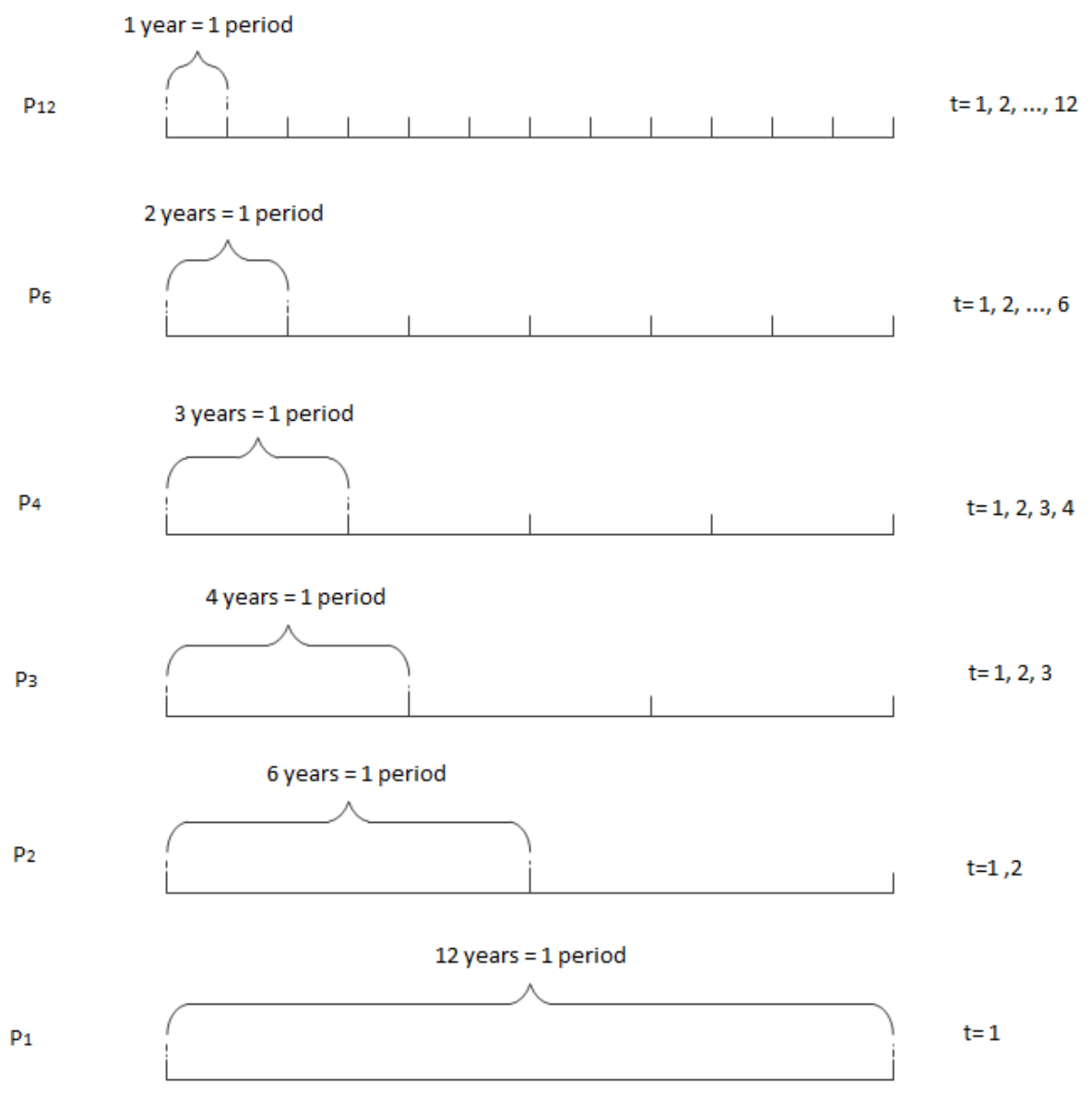

Figure 4.8: Problems with Different Period Lengths

Figure 4.8 shows how these problems are generated. Regardless of the period lengths, the mathematical formulation remains the same, but data changes according to the number of years in a period. As mentioned before, demand and cost data are generated for each year in the planning horizon. Based on these data, yearly demands are aggregated to obtain periodical demand in each problem. For example, for 1period problem, demand values of 12 years are combined while in 2-period problem, demand data of the first period is the summation of the first 6 years' demand values. 
As in demand data, operating costs of compressor stations and pipelines are aggregated according to the period length. However, investment, transportation, and purchase costs in each period represents the cost of corresponding beginning year of the period. For example, in 1-period problem, operating costs are the summation of operating costs in all 12-year span, and the investment costs are the first year's costs. In the same manner, for 2-period problem, the summation of operating costs of each 6-year span gives the operating cost of one period. The investment cost of each period in a 2-period problem is the cost in the corresponding beginning year. More precisely, in the first 6-year span, the costs are the same as the costs in the beginning year while in the second 6 year span, they are equal to the costs in the seventh year.

Supply contracts will be made at the beginning of each time span so purchase cost will be charged in each period. For example, for a 12 period problem gas will be purchased yearly. Purchase cost vary for each period because it is assumed that gas prices increase $2 \%$ each year. For 1-period problem, gas for the whole 12 years will be purchased in the first period at the rate in the first year. The total costs of different period lengths vary due to these cost variations.

1-period problem implies that the planning decisions made in that period will cover 12 years. Pipe and compressor arcs used in the first period will be available for 12 years. 2-period problem gives solutions for each 6-year span in the whole planning horizon. The first period contains solutions for the first 6 years, while the second period represents the next 6 years. Any changes occur in a period will be available in the corresponding 6 years. In a 12-period problem, the solution contains planning decisions for each year independently, so in each year a change may occur. 


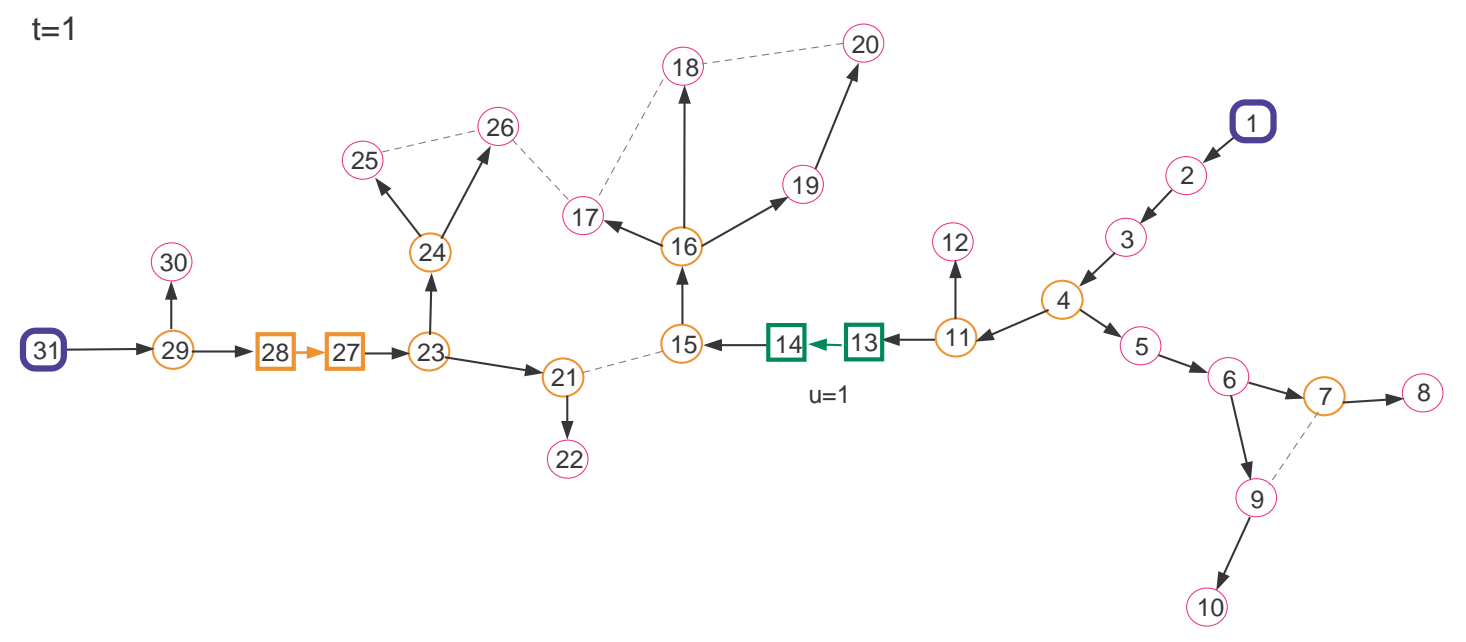

Figure 4.9: Solution to the 1-Period Planning

The network structure that is shown in Figure 4.9 is the solution to the 1-period problem. The network consists of two trees with 26 pipe arcs. The compressor stations at the $13-14$ nodes and 27-28 nodes are active. Pressure at the node 28 is above 45 bar, but the model installs compressor station at that node in order to balance the pressure drop and flow rate. The net present value of the network is $\$ 82,140,847,000$. This problem provides the maximum minimized cost because the model does not allow any changes in 12 years. The current network will be available for the whole planning horizon. 

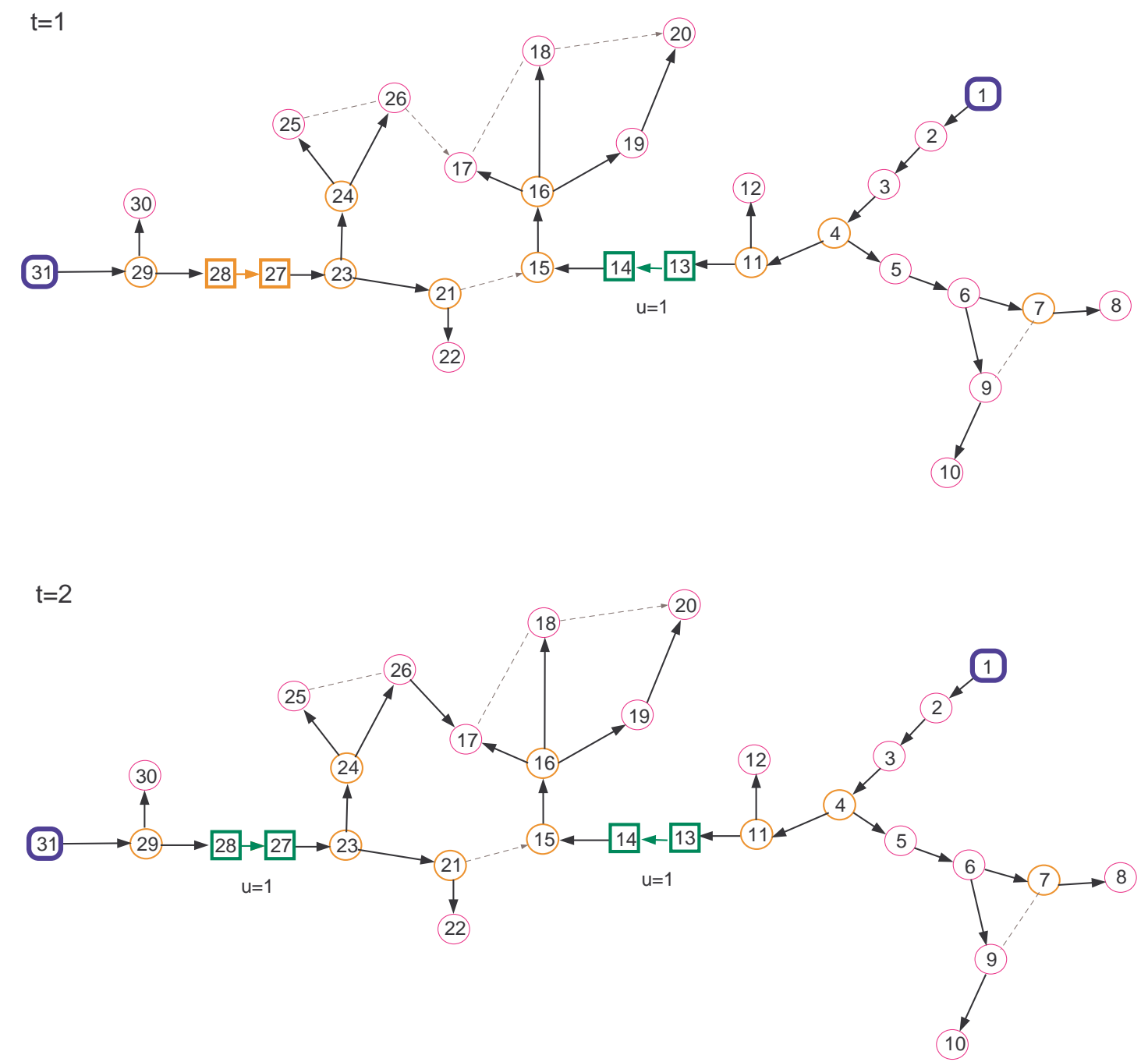

Figure 4.10: Solution to the 2-Period Planning

The solution to the 2-period problem is shown in Figure 4.2.2 and Figure 4.10. The network remains the same in two 6 year spans. In the first period, a compressor station at nodes 13-14 will be installed, and it will be available for the whole planning horizon. The network structure is the same as the solution to the 1-period planning problem in the second period. Compressor station at nodes 27-28 will be active starting from the second 6 year span. The cost is less for this 2-period problem 
because of transportation cost, and the investment and operating costs of pipelines and compressor stations, in two periods. Furthermore, the total flow rate in a period varies depending on the period length so the total cost varies between planning horizon lengths.
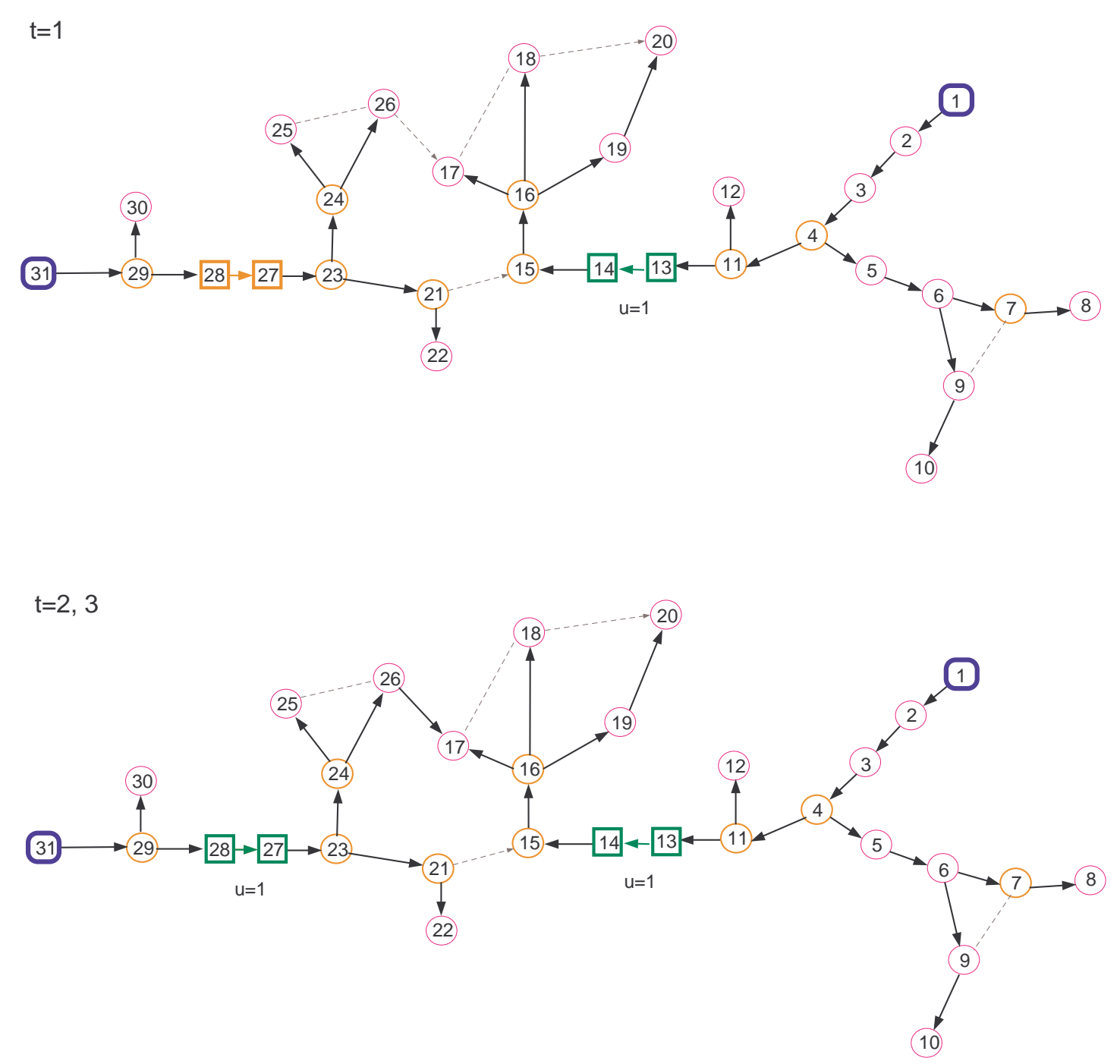

Figure 4.11: Solution to the 3-Period Planning 
As shown in Figure 4.2.2 and Figure 4.11, for the first 4 year span of the 3-period planning problem, the network structure is the same as the first periods of problems with greater period length. For the last two periods, more precisely, in the last 8 years of the planning horizon, new pipeline and a compressor station at nodes 27-28 will be installed.
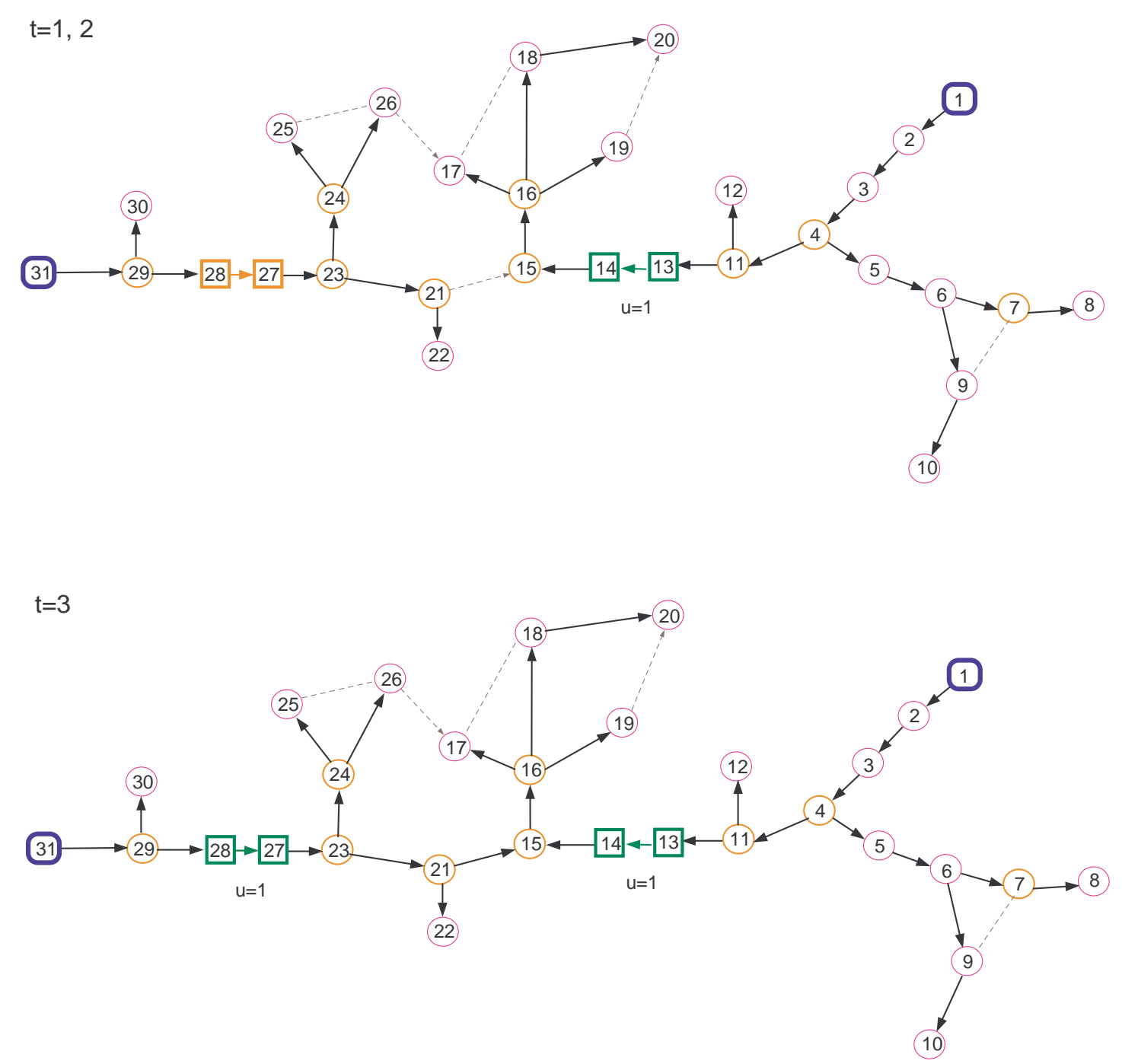

Figure 4.12: Solution to the 4-Period Planning (1) 


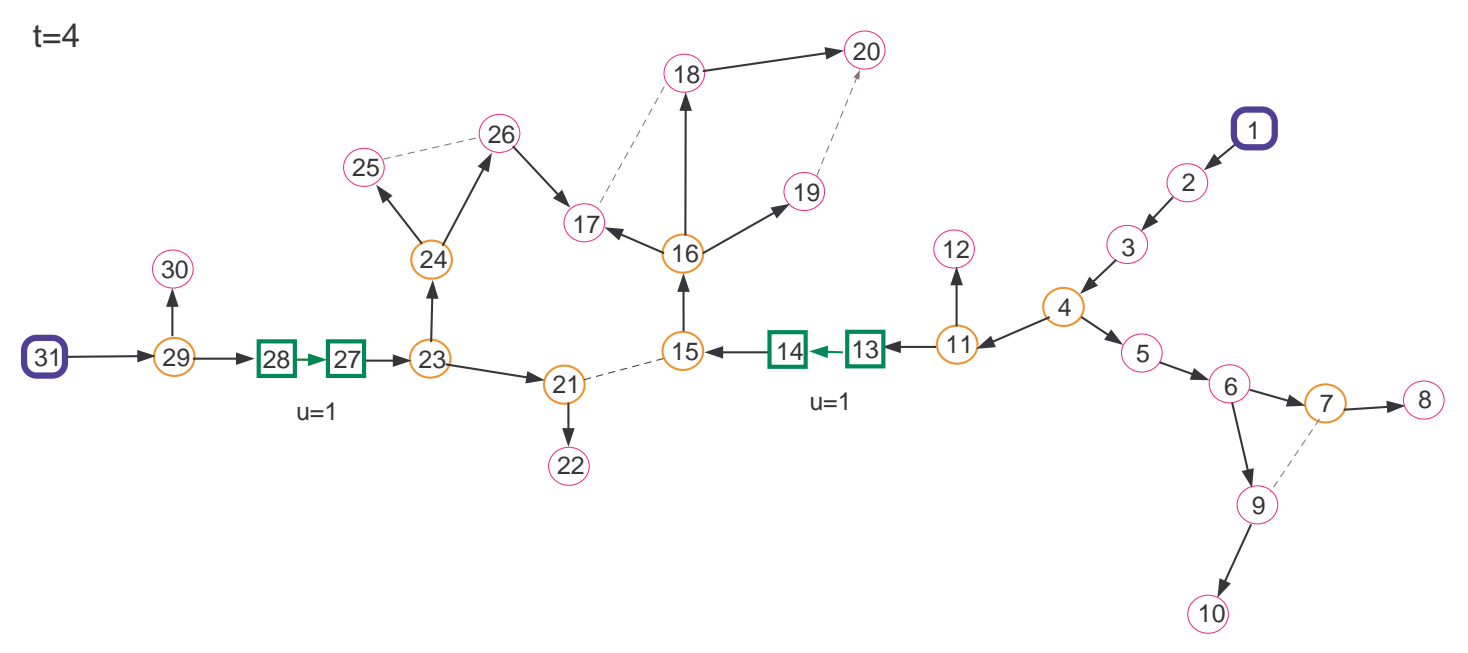

Figure 4.13: Solution to the 4-Period Planning (2)

As shown in Figures 4.2.2, 4.12, and 4.13 in the 4-period planning, there are three structures throughout 12 years. Compressor station at node 27-28 are available starting from the third 3 year span. In the 2-period problem, these nodes are also active starting from seventh year, while in 3-period problem they are active starting from the fifth year. Even though changes in the network are compatible for different period lengths, costs are different. The effects of the depreciation, investment and operating costs on the flow rate increase with the decrease in period lengths so the model tries to minimize the total flow rate by using different pipe arcs over 4 period problem. Therefore, variations on the network structure over the planning horizon can be seen more clearly in short period lengths. 

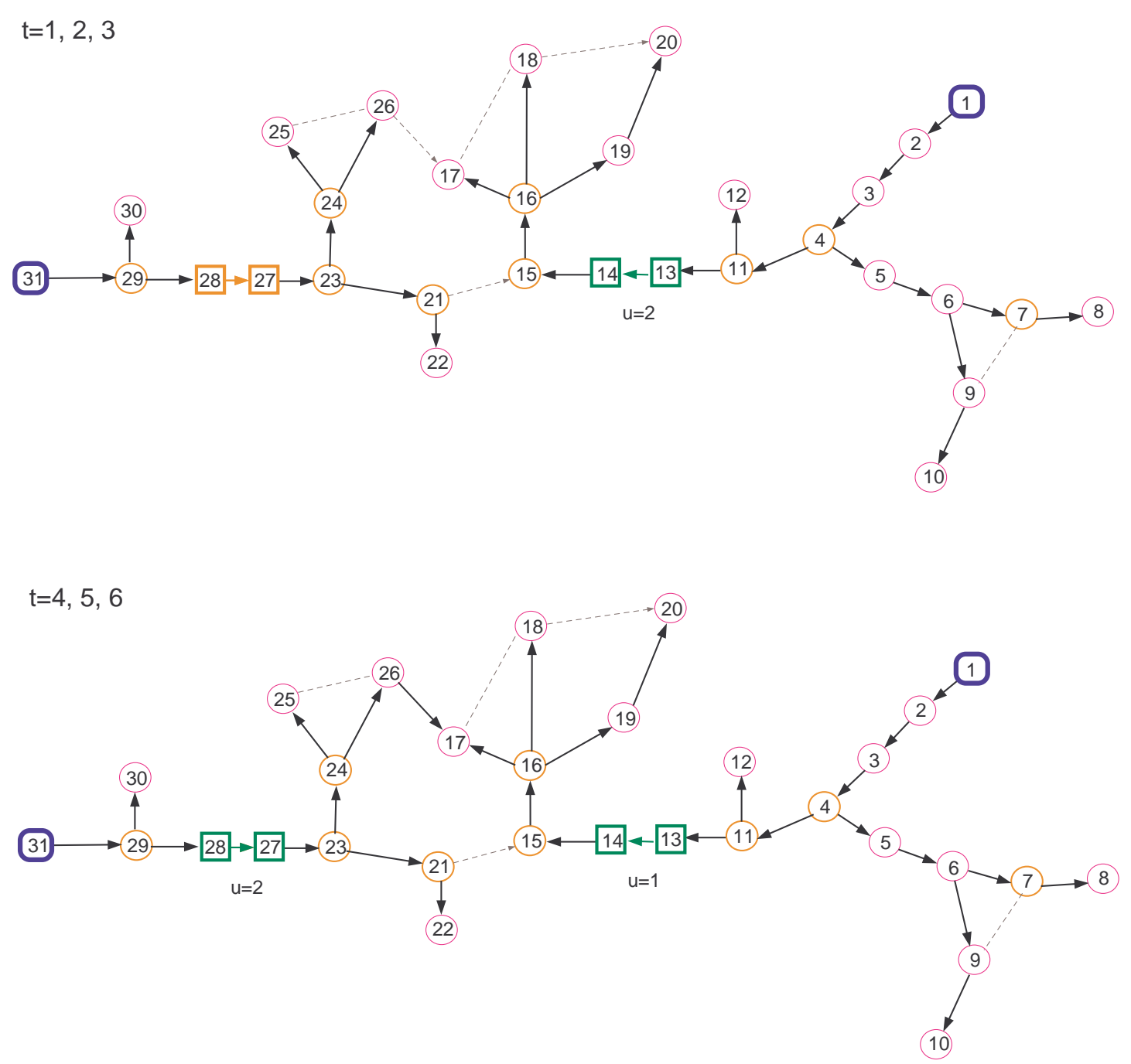

Figure 4.14: Solution to the 6-Period Planning

Figure 4.2.2 and Figure 4.14 represent the solution to the 6-period planning. The network consists of only one tree and the compressor station at nodes 13-14 is active while the one at nodes $27-28$ is bypassed in the first 3 periods. As in the previous problems, both compressor stations are active in the latter periods because of the increasing demand. The model tries to keep the balance between the flow rate and the pressure drop. Therefore, in the last 3 periods, the model requires installation of 
a compressor station at nodes 27-28 starting from the seventh year to increase the pressure. As in the previous problems, there are two pipe arcs that deliver gas to node 17 . To minimize the total flow, the model installs new pipeline from node 26 to node 17 . Thus, both the flow rate and the cost is minimized.

$t=1,2,3,4,5,6$

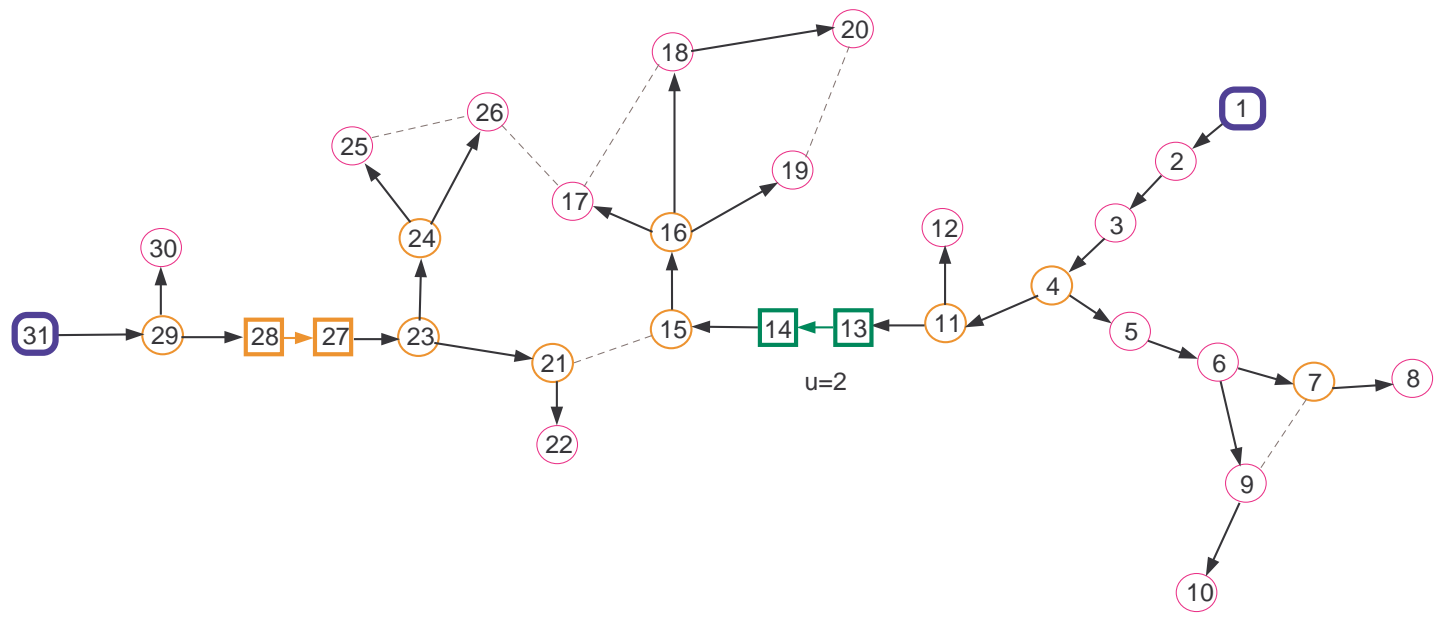

$\mathrm{t}=7,8,9,10,11,12$

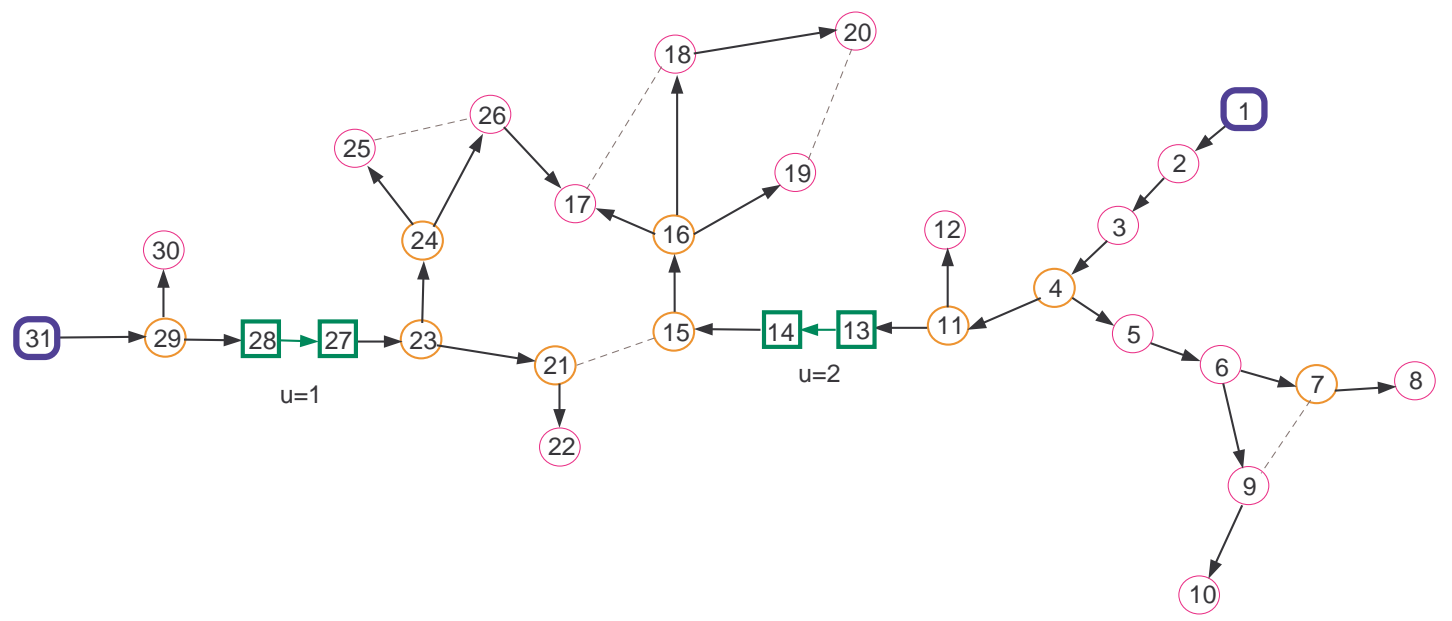

Figure 4.15: Solution to the 12-Period Planning 
Figure 4.2.2 and Figure 4.15 show the output of the 12-period planning. The model gives the optimal solution by minimizing the total cost of each period. Since, the 12-period problem output includes solutions for each year, it provides the least cost among the other problems. The total cost of the network in 12 years is $\$ 79,182,330,000$.

Only one compressor station is used in the first six periods while both of them are active in the latter periods.

The optimal total cost and the solution time of each problem instance are given in Table 4.5.

Table 4.5: Optimal Costs and Solution Times for Different Period Lengths

\begin{tabular}{|c|c|c|r|r|r|}
\hline Problem & $\begin{array}{c}\text { Number of } \\
\text { Periods }\end{array}$ & $\begin{array}{r}\text { Period } \\
\text { Length }\end{array}$ & $\begin{array}{r}\text { Best Objective } \\
\text { Value }(\$)\end{array}$ & $\begin{array}{r}\text { CPU Time } \\
\text { (second) }\end{array}$ & Gap \% \\
\hline$P_{1}$ & 1 & 12 years & $82,140,847,000$ & 47.37 & 0.0013 \\
$P_{2}$ & 2 & 6 years & $81,297,629,000$ & 174.91 & 0.0031 \\
$P_{3}$ & 3 & 4 years & $80,540,463,000$ & 3436.85 & 0.00 \\
$P_{4}$ & 4 & 3 years & $79,781,491,000$ & 4956.32 & 0.00066 \\
$P_{6}$ & 6 & 2 years & $79,418,565,000$ & 16352.65 & 0.00022 \\
$P_{12}$ & 12 & 1 year & $79,182,330,000$ & 21351.74 & 0.00024 \\
\hline
\end{tabular}

As a result of this experiment, it can be concluded that;

- In the Table 4.5, it can be observed that, while the number of periods increase, the total cost decreases, because changes in the network that are made in a period will be available for years the period covers. For a 1-period problem, if a compressor station is installed, the operating cost will be charged for 12 years. However, for a 12-period problem, a compressor station may not be needed in the first 6 years so the operating cost will be paid starting from the 
seventh period. The operating cost for pipeline is also charged in the same way. Therefore, solving each period individually helps with making the right decisions in the right time.

- The key factor of the differences between the various planning horizons is the strategy that is used to compute demand and cost for each one. For example, for 6-period planning, it is assumed that demand values are the summation of two consecutive years, while for 1-period planning demand values are the summation of all yearly demands in 12 years. Demand values define the daily flow rate in a pipeline. Since, demand values change in each year, daily flow rates are also subject to change. Compressor stations are located at nodes according to the pressure. Therefore, pressure values at nodes as well as the location of compressor stations, are different in each year due to the changes in daily flows. Moreover, since transportation and purchase costs depend on the flow rate, the total cost also varies.

- Another factor is that the model allows changes in the network during long time periods. For example, in 12-period planning, the network is allowed to change during each of the 12 years to operate with the minimum cost. Thus, the network changes after the sixth period. In the same manner, 4-period planning gives 3 different network structure. The model makes changes on the network to minimize the total cost. However, in 1-period planning, it is assumed that the network will remain the same for 12 years as if it is in the first period. On the other hand, the network structure may not be convenient for the latter periods, so there can be sunk costs. Therefore, the cost increases while the period length decreases. 
- Demand values and cost data are crucial parameters for the NG network. Therefore, the aggregate planning may not give reliable solutions to NG transmission networks over long planning horizons.

- This study shows that planning each year independently from other years gives more realistic solution with minimal costs. By using a multi-period planning problem, compressor station and pipeline installation decisions can be made more accurately.

In this experiment, it is shown that very similar network structure can be obtained by using different period lengths for a planning horizon. The annual flow costs implied by the problems, $P_{1}, P_{2}, \ldots, P_{12}$, of each scenario are compared to show the robustness of the solution. To obtain associated flow cost for each problem, transportation, and purchase costs are calculated for each year separately. The network structures are fixed as obtained in each case and individual flow problems are solved by using corresponding networks. Thus, it is assumed that these networks are existing networks and they will be available as long as the period length of a scenario. For example, for 1-period problem, network decisions for the whole planning horizon are made only once at the beginning, so the network structure remains the same during the whole planning horizon. The values of cost parameters, which are transportation and purchase costs, of the first year are used for each year. Since the cost data is the same in each year, the annual total cost varies over 12 years according to the total flow. However, in 2-period problem, the network structure is modified after sixth year, so the total flow changes. The cost parameter values also vary in every two years due to gas prices or electricity costs. Therefore, yearly costs vary over 12 years depending on both the cost and flow. Other problems Figure 4.16 shows the annual transportation and purchase costs of each problem. 


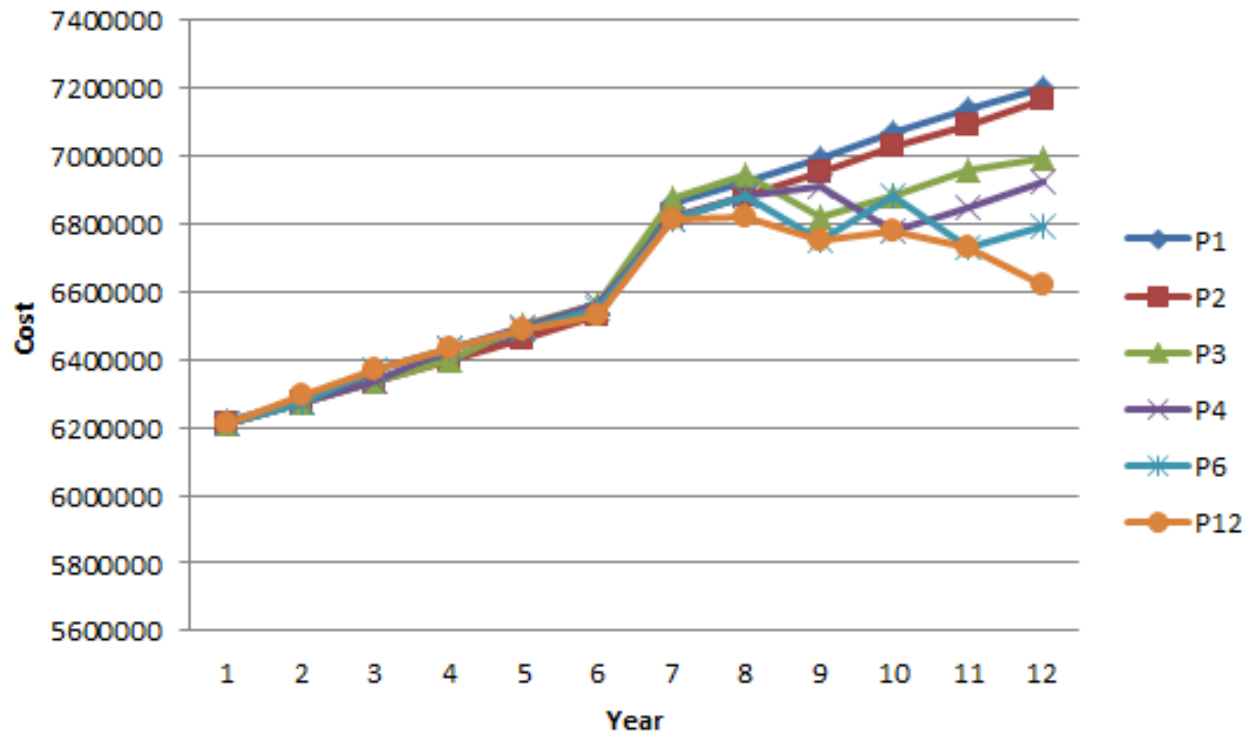

Figure 4.16: Annual Costs of Different Period Lengths

As shown in Figure 4.16, for the first 7-year-span, the annual total costs of all problems are very close to each other. Therefore, for a decision making process, computational times of the problems must be considered. Consequently, 1-period problem can be chosen, since it gives optimum results in a short time. However, the total cost of the problem is not a minimum cost that can be obtained in 12 years. The problem also assumes that there is not any change in the network structure during planning horizon. Therefore, to obtain a realistic network structure and modifications with a minimum cost, 12-period problem can be used.

For the rest of the planning horizon, the total costs vary depending on the period lengths. As mentioned before, 1-period problem network structure does not change during planning horizon, but demand increases over time. Therefore, even though the cost parameter values are the same for all years, the total cost increases because of the increase in flow. However, the output of the 12-period problem allows changes 
in the network structure due to increasing demand. For example, if a pipeline is needed in sixth year, 1-period problem may install it in the first year or it may not consider it at all. The other problems that include more periods react the change in time and adjust the network accordingly. Another advantage of 12-period planning is the cost variation over planning horizon. The transporting cost of one mmscm of gas decreases during 12 years while the total flow increases. Therefore, the total cost is less than the total cost of 1-period problem. Thus, the difference between costs increases over the last 5 years because of the cost variation in the problems.

The model can be run in different periods of time by using updated data. For example, if new demand forecasts are available after sixth year, the model can be used to optimize flow to find the minimum amount of gas purchase in the next periods. Moreover, for existing networks, by fixing variables corresponding to pipeline and compressor station arcs, expansion decisions, such as installing new pipelines or compressor stations, can be made.

In summary, the operational and strategic plan for 12 years can be made at the beginning of the planning horizon using a 1-period model structure, which has the least computational time. However, since demand increases, the network may require new pipelines and compressor stations over time; 1-period problem may not give efficient solution for the next periods. 12-period problem gives reliable solutions, but the computational time is more than the other problems. Consequently, considering both the computational time and the output efficiency, the problems with $2,3,4$ or 6 periods can be used. Thus, the model can be run in each period to make decisions for the latter periods. 


\subsubsection{Experiment 3: The Effect of Changes in the Cost Parameters}

In this experiment, the model performance with different cost parameters is examined. Another purpose of the study is to find the cost that affects the network structure most. For this purpose, various test instances are generated for the 31-node 12-period network. The cost of investment and of operating compressor stations are treated as one parameter. In the same manner, the total cost for pipelines consists

of the investment and operating costs. Also, the transportation cost is taken into consideration to observe the changes in the network due to variable cost parameters. Thus, the three costs used in the test runs are the total cost of compressor stations, the total cost of pipelines, and the transportation cost. The upper and lower bounds of the costs are computed by using $20 \%$ of each value. Smaller percentages of the cost parameters did not affect the network remains the same in test runs of the model, but for $20 \%$ and up the network structure changes.

The cost data that are used in these instances are shown in Table 4.6 and 4.7.

Test instances include different combinations of the lower and upper values of costs. These combinations are shown as (I-II-III), where the first entry refers to the transportation cost, the next one represents the total cost of pipelines and the last one stands for the total cost of compressor stations. For example, (L-U-L) implies that the problem has the lower values of transportation cost and the total compressor station costs, and the upper value of the total pipeline cost. The best objective function values and the solution times of instances are shown in Table 4.8. 
Table 4.6: Cost Data $\left(10^{3} \$\right)(1)$

\begin{tabular}{|c|c|c|c|c|c|c|c|c|c|}
\hline Cost & Period & Actual Cost & Lower & Upper & Cost & Period & Actual Cost & Lower & Upper \\
\hline \multirow{12}{*}{$\alpha_{t}^{c}$} & 1 & 10243.865 & 8195.092 & 12292.638 & \multirow{12}{*}{$\beta_{t}^{c}$} & 1 & 24.709 & 19.7672 & 29.6508 \\
\hline & 2 & 9878.216 & 7902.5728 & 11853.8592 & & 2 & 25.944 & 20.7552 & 31.1328 \\
\hline & 3 & 9459.014 & 7567.2112 & 11350.8168 & & 3 & 27.242 & 21.7936 & 32.6904 \\
\hline & 4 & 8978.674 & 7182.9392 & 10774.4088 & & 4 & 28.604 & 22.8832 & 34.3248 \\
\hline & 5 & 8428.558 & 6742.8464 & 10114.2696 & & 5 & 30.034 & 24.0272 & 36.0408 \\
\hline & 6 & 7798.837 & 6239.0696 & 9358.6044 & & 6 & 31.536 & 25.2288 & 37.8432 \\
\hline & 7 & 7078.328 & 5662.6624 & 8493.9936 & & 7 & 33.112 & 26.4896 & 39.7344 \\
\hline & 8 & 6254.309 & 5003.4472 & 7505.1708 & & 8 & 34.768 & 27.8144 & 41.7216 \\
\hline & 9 & 5312.313 & 4249.8504 & 6374.7756 & & 9 & 36.506 & 29.2048 & 43.8072 \\
\hline & 10 & 4235.891 & 3388.7128 & 5083.0692 & & 10 & 38.332 & 30.6656 & 45.9984 \\
\hline & 11 & 3006.347 & 2405.0776 & 3607.6164 & & 11 & 40.248 & 32.1984 & 48.2976 \\
\hline & 12 & 1602.431 & 1281.9448 & 1922.9172 & & 12 & 42.261 & 33.8088 & 50.7132 \\
\hline \multirow{12}{*}{$\alpha_{t}^{p}$} & 1 & 109.297 & 87.4376 & 131.1564 & \multirow{12}{*}{$\beta_{t}^{p}$} & 1 & 21.859 & 17.4872 & 26.2308 \\
\hline & 2 & 105.396 & 84.3168 & 126.4752 & & 2 & 21.079 & 16.8632 & 25.2948 \\
\hline & 3 & 100.923 & 80.7384 & 121.1076 & & 3 & 20.185 & 16.148 & 24.222 \\
\hline & 4 & 95.798 & 76.6384 & 114.9576 & & 4 & 19.16 & 15.328 & 22.992 \\
\hline & 5 & 89.929 & 71.9432 & 107.9148 & & 5 & 17.986 & 14.3888 & 21.5832 \\
\hline & 6 & 83.21 & 66.568 & 99.852 & & 6 & 16.642 & 13.3136 & 19.9704 \\
\hline & 7 & 75.522 & 60.4176 & 90.6264 & & 7 & 15.104 & 12.0832 & 18.1248 \\
\hline & 8 & 66.73 & 53.384 & 80.076 & & 8 & 13.346 & 10.6768 & 16.0152 \\
\hline & 9 & 56.68 & 45.344 & 68.016 & & 9 & 11.336 & 9.0688 & 13.6032 \\
\hline & 10 & 45.195 & 36.156 & 54.234 & & 10 & 9.039 & 7.2312 & 10.8468 \\
\hline & 11 & 32.076 & 25.6608 & 38.4912 & & 11 & 6.415 & 5.132 & 7.698 \\
\hline & 12 & 17.097 & 13.6776 & 20.5164 & & 12 & 3.419 & 2.7352 & 4.1028 \\
\hline
\end{tabular}


Table 4.7: Cost Data $\left(10^{3} \$\right)(2)$

\begin{tabular}{|c|c|c|c|c|}
\hline Cost & Period & Actual Cost & Lower & Upper \\
\hline \multirow{6}{*}{} & 1 & 20.95 & 16.76 & 25.14 \\
& 2 & 20.21 & 16.16 & 24.25 \\
& 3 & 19.35 & 15.48 & 23.22 \\
& 4 & 18.37 & 14.69 & 22.04 \\
& 5 & 17.24 & 13.79 & 20.69 \\
& 6 & 15.95 & 12.76 & 19.14 \\
& 7 & 14.48 & 11.58 & 17.38 \\
& 8 & 12.80 & 10.24 & 15.36 \\
& 9 & 10.87 & 8.70 & 13.04 \\
& 10 & 8.67 & 6.94 & 10.40 \\
& 11 & 6.15 & 4.92 & 7.39 \\
& 12 & 3.28 & 2.63 & 3.94 \\
\hline
\end{tabular}

Table 4.8: Optimal Costs and Solution Times for Different Combinations of Cost Parameters

\begin{tabular}{|c|c|c|c|c|}
\hline Problem & $\begin{array}{c}\text { Test } \\
\text { Instance }\end{array}$ & $\begin{array}{c}\text { Best objective } \\
\text { value (\$) }\end{array}$ & $\begin{array}{c}\text { CPU time } \\
\text { (second) }\end{array}$ & Gap \% \\
\hline$T_{0}$ & A-A-A & $79,182,330,000$ & 21351.74 & 0.00024 \\
$T_{1}$ & L-L-L & $77,488,495,000$ & 21764.74 & 0.00189 \\
$T_{2}$ & L-L-U & $77,502,091,000$ & 17187.34 & 0.00204 \\
$T_{3}$ & L-U-L & $77,576,231,000$ & 22684.36 & 0.00258 \\
$T_{4}$ & L-U-U & $77,622,053,000$ & 19981.68 & 0.00277 \\
$T_{5}$ & U-L-L & $80,253,805,000$ & 19728.26 & 0.00354 \\
$T_{6}$ & U-U-L & $80,317,453,000$ & 19628.51 & 0.00358 \\
$T_{7}$ & U-L-U & $80,336,401,000$ & 19378.53 & 0.00439 \\
$T_{8}$ & U-U-U & $80,449,709,000$ & 19609.75 & 0.00492 \\
\hline A: Actual cost / L: Lower value / U: Upper Value \\
\hline \multicolumn{5}{|l}{}
\end{tabular}


Flow rate variables determine the design of the network. A pipeline is installed between nodes $i$ and $j$, if gas flows between these nodes. Therefore, the transportation cost that is related to the flow rate determines the network design. For the lower values of the transportation cost, the network structure is the same as the original 12-period network. Different values of the total costs of compressor stations and pipelines do not affect the network. As mentioned before, the model tries to find the minimized total flow rate to reduce the costs. If transportation cost values are high, the model searches for new paths to transport gas to keep the total flow rate at a minimum so there are various structure throughout 12 years for each problem.

Figure 4.17 shows the solution to the problem with the upper values of transportation cost and the lower values of compressor station costs. The network remains the same for the lower and upper values of pipeline costs. Figure 4.18 represent the problem output for the upper values of the transportation cost and compressor station costs. This network is also the same for the different values of pipeline costs. 

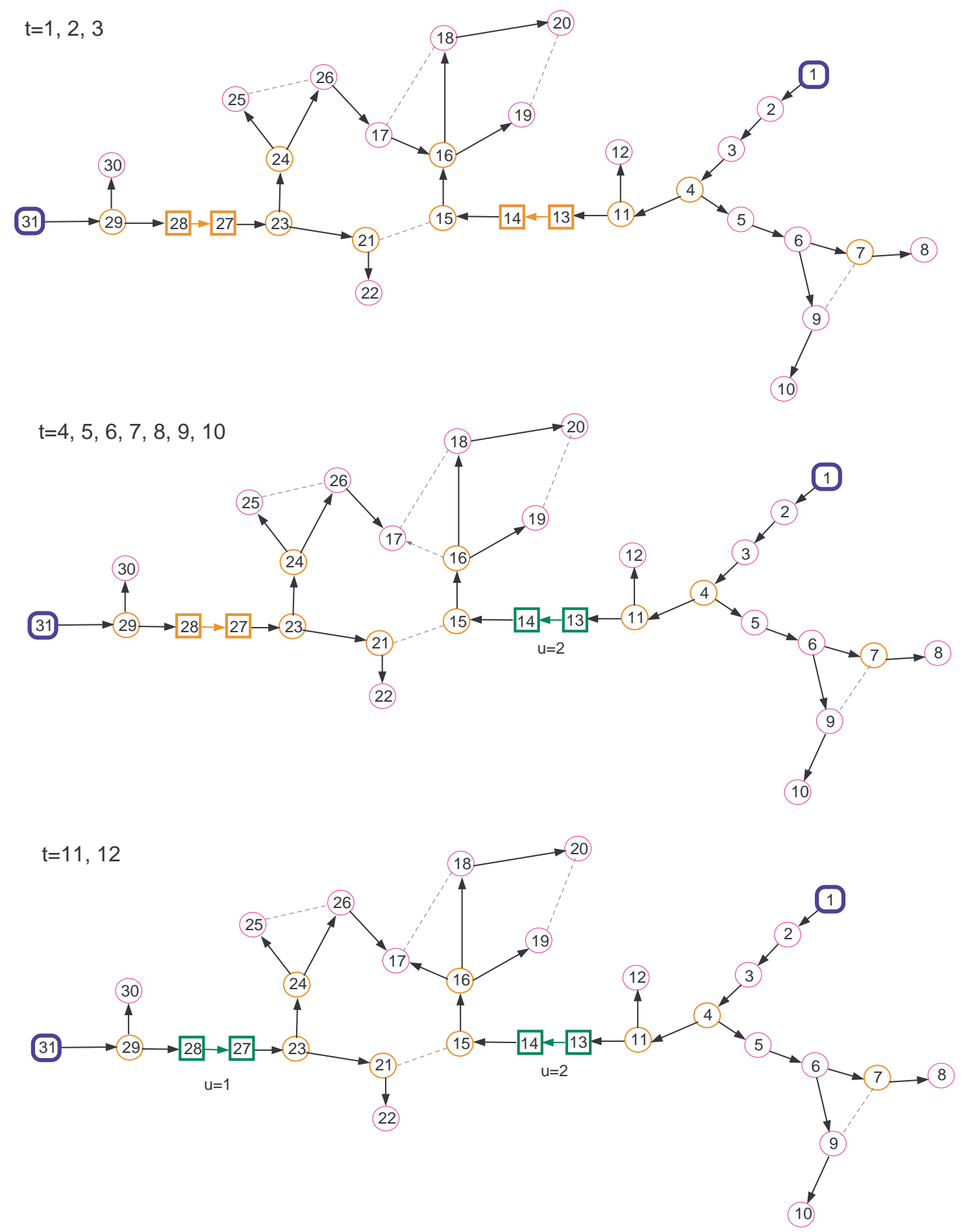

Figure 4.17: Network Structure for Problems $T_{5}$ and $T_{6}$ 

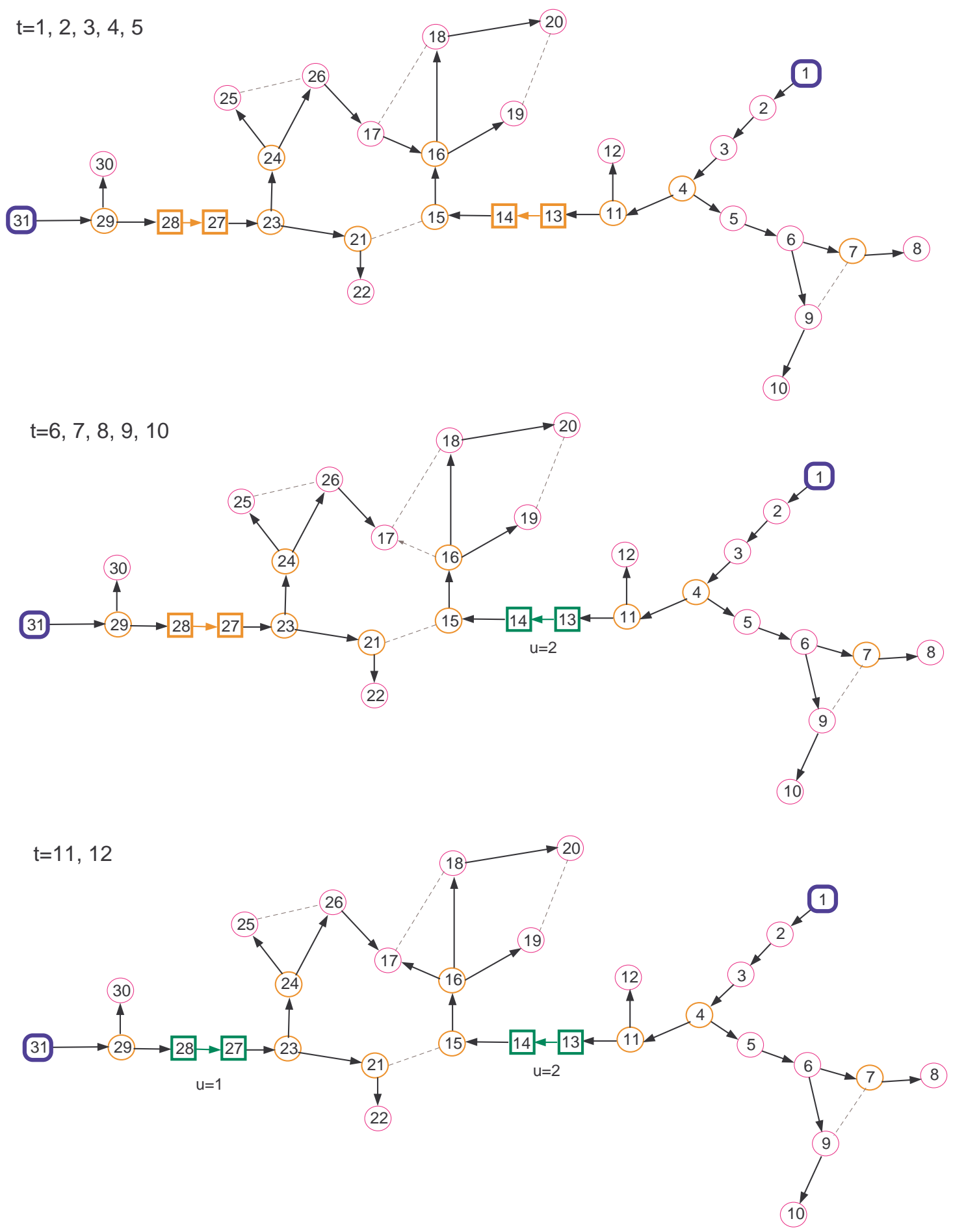

Figure 4.18: Network Structure for Problems $T_{7}$ and $T_{8}$ 
As shown in the Figure 4.17 and Figure 4.18, for the upper values of the transportation cost, the network structure changes according to the total costs of compressor stations. If the total cost of compressor stations is at the lower value then the model installs a compressor station at nodes 13-14 starting from the fourth period. If it is at the upper value then the station becomes active in the sixth period. The costs of the pipeline do not change the structure.

In summary, it can be concluded that transportation cost is the most important cost that affects the network structure. Decisions for choosing the best transportation cost parameter must be made carefully. Too low or too high values can change the network completely. Thus, the solution may not be applicable to the real-world system. This study also showed that the model built with the upper values of transportation cost and compressor stations tries to delay the installation decisions for compressor stations. If installing compressor stations in the latter periods is compatible with the other long-term plans, and the costs of the network are also convenient for the company budget, then system analysts can use make decisions accordingly. Since the model is cost sensitive, the scale between the cost parameters is important. Costs must be calculated carefully to avoid getting any inapplicable and costly results. 


\section{CONCLUSIONS AND FUTURE RESEARCH DIRECTIONS}

This research addresses the problem of the optimization of a steady-state NG transmission network. A MINLP model was proposed for minimizing the total investment and operating costs. The main contribution of this study is to provide an integrated optimization model consisting of decision variables and constraints that other researches studied before separately. The systematic design of various test instances produced in this study assessed the proposed optimization approach, when applied to different sized problems, in terms of efficiency and reliability. The economic aspects of NG pipeline transportation were reviewed. Concepts of the investment cost of pipeline and compressor stations and the periodic operating costs were introduced. The problem was modeled with AMPL. The numerical experiments were conducted by application of a MINLP, Bonmin. This model will assist decision makers to make appropriate decisions within a short time.

Flow networks have a complex structure in regard to gas characteristics and any change or modification of their structure while in use is costly and difficult. Therefore, designing a new network and utilizing of its capabilities optimally is important. In the network model, arcs correspond to pipelines and compressor stations. The pipeline flow and gas pressure at each node are the main decision variables in the problem. In

addition to flow conservation constraints, the model also includes a constraint that defines the relationship of flow rate and pressure.

In the first type of test problems, the model was tested on three different networks with 31, 66 and 97 nodes by using data for 1 period. In these problems, the actual investment and operating costs were used. Demand parameters were representative of the real-world system. Then, by generating different parameter settings, the changes 
on the network structure were evaluated. For the second type of test problems, the lengths of the periods were varied in order to detect the different modifications that may occur in the system due to changes in the variables related to long-lasting decisions. The robustness of the model output under varying scenarios was tested. In the third type of test problems, discrete intervals for the cost parameter values were used. The model was updated for each cost parameter value with different intervals and the results were compared. The aim of this analysis is to assess the effect of cost changes on the network structure as well as the run time of the problems. These tests and analyses were applied on a small-size network with 31 nodes.

These test runs indicated that the proposed model gives effective results for multiperiod planning problems. The changes in the network structure can be observed, and strategic and operational decisions can be made accordingly. The model provides solutions with minimized costs by reducing the total flow rate during a period. This strategy requires fewer changes in the network over planning horizon. Since compressor stations and pipelines are long-term investment decisions, system analysts try to maintain the same network for long years to avoid sunk costs. They also try to minimize the number of idle pipelines and compressor stations in a period to maximize the system efficiency. The solutions to the test instances showed that the proposed model minimizes the total cost by making fewer changes on the network for multi-period problems to satisfy increasing demand over planning horizon.

There are a couple of assumptions and limitations of this study. First, penalty costs were not included in the study. In real-world problems, if the amount of consumption is below the supply contract amount in a period, then the cost of penalty must be paid for the excess amount of supply. Second, it is assumed that there is no NG storages in the system. Gas is delivered only from different suppliers. The first limitation may be overcome by introducing a new variable representing 
the excess mount of supply. A new constraint may be defined to add the storage to the system. The proposed model allows defining new variables and constraints easily to enhance the interest area. In the operating perspective, there could be other objective functions of interest such as minimizing the fuel consumption at compressor stations.

In this study, optimization of design and operation of the steady-state natural gas transmission systems were studied. Findings about the multi-purpose mathematical model can be transferable to a wide range of research areas. 


\section{REFERENCES}

[1] Arsegianto, A., E. Soewono, M. Apri. 2003. Non-linear optimization model for gas transmission system: A case of grissik - duri pipeline. Asia Pacific Oil and Gas Conference and Exhibition, Society of Petroleum Engineers.

[2] Belotti, P. 2006. COUENNE: a users manual. URL http: //www. coin-or.org/Couenne.

[3] Belotti, Pietro. 2012. Disjunctive cuts for nonconvex minlp. Mixed Integer Nonlinear Programming, The IMA Volumes in Mathematics and its Applications, vol. 154. Springer, New York, New York, 117-144.

[4] Bonami, P., J. Lee. 2011. Bonmin user's manual. URL http: //www. coin-or.org/Bonmin.

[5] Borraz-Sanchez, C. Oct. 2010. Optimization methods for pipeline transformation of natural gas. Ph.D. Dissertation, University of Bergen, Norway .

[6] Borraz-Sanchez, C., R. Rios-Mercado. 2009. Improving the operation of pipeline systems on cyclic structures by tabu search. Computers and Chemical Engineering 33 58-64.

[7] Boyd, S., A. Ghosh, A. Magnani. 2003. Branch and bound methods. URL Notes for EE392o, Stanford University.

[8] Chebouba, A., F. Yalaoui, A. Smati, L. Amodeo, K. Younsi, A. Tairi. 2009. Optimization of natural gas pipeline transportation using ant colony optimization. Computers $\&$ Operations Research 36 1916-1923. 
[9] Chung, T.S., K.K. Li, G.J. Chen, J.D. Xie, G.Q. Tang. 2003. Multi-objective transmission network planning by a hybrid ga approach with fuzzy decision analysis. International Journal of Electrical Power \& Energy Systems 25 187192.

[10] Czyzyk, J., M. Mesnier, J. More. 1998. The NEOS server. IEEE Journal on Computational Science and Engineering 5 68-75.

[11] De Wolf, D., Y. Smeers. 2000. The gas transmission problem solved by extension of the simplex algorithm. Management Science 46 1454-1465.

[12] Fourer, R., David M. Gay, Brian W. Kernighan. 2002. AMPL: A Modeling Language for Mathematical Programming. Duxbury Press, Pacific Grove, California.

[13] Hamedi, M., R.Z. Farahani, M.M. Husseini, G.R. Esmaeilian. 2009. A distribution planning model for natural gas supply chain: a case study. Energy Policy 37 799-812.

[14] Hamedi, M., R.Z. Farahani, M.M. Husseini, G.R. Esmaeilian. 2011. Optimization in natural gas network planning. Logistics Operations and Management : Concepts and Models - 393-420.

[15] Kabirian, A., M. R. Hemmati. 2007. A strategic planning model for natural gas transmission networks. Energy Policy 35 5656-5670.

[16] Kennedy, John L. 1993. Oil and Gas Pipeline Fundamentals. PennWell Books, Tulsa, Oklahoma.

[17] Lawler, E. L., D. E. Wood. 1966. Branch-and-bound methods: A survey. Operations Research 14 699-719. 
[18] Lougee-Heimer, R. 2003. The common optimization interface for operations research: Promoting open-source software in the operations research community. IBM J. Res. Dev. 47 57-66.

[19] MATLAB. 2010. version 7.10.0 (R2010a). The MathWorks Inc., Natick, Massachusetts.

[20] Menon, Shashi E. 2005. Gas Pipeline Hydraulics. CRC Press, Boca Raton, Florida.

[21] Osiadacz, A. J., D. J. Bell. 1986. A simplified algorithm for optimization of large-scale gas networks. Optimal Control Applications and Methods 7 95-104.

[22] Pratt, K.F., J.G. Wilson. 1984. Optimization of the operation of gas transmission system. Transaction of the Instrument of the Measurement and Control 19 $261-269$.

[23] Rios-Mercado, R., S. Kim, E. Boyd. 2006. Efficient operation of natural gas transmission systems: A network-based heuristic for cyclic structures. Computers \& Operations Research 33 2323-2351.

[24] Rios-Mercado, Roger Z., S. Wu, L. Ridgway Scott, E. Andrew Boyd. 2001. A reduction technique for ng transmission network optimization problems. Annals Of Operations Research 117 217-234.

[25] Rosenthal, Richard E. 2012. GAMS: A user's guide. URL http: //www . gams . com/.

[26] Sahinidis, N., M. Tawarmalani. $2011 . \quad$ BARON. URL http: //www gams. com/dd/docs/solvers/baron.pdf. 
[27] Tabkhi, F. Dec. 2007. Optimisation de réseaux de transport de gaz. Ph.D. Dissertation, Institut National Polytechnique de Toulouse, France .

[28] Uraikul, V., C.W. Chan, P. Tontiwachwuthikul. 2003. MILP model for compressor selection in natural gas pipeline operations. International Society for Environmental Information Sciences, Environmental Informatics Archives 1138 145 .

[29] US Department Of Energy, Energy Information Administration. 2009. International energy annual 2009. http://www.energy.gov .

[30] US Department Of Energy, Energy Information Administration. 2010. International energy annual 2010. http://www.energy.gov .

[31] Woldeyohannes, Abraham D., Mohd A. A. Majid. 2011. Simulation model for natural gas transmission pipeline network system. Simulation Modelling Practice and Theory 19 196-212.

[32] Wong, P. J., R. E. Larson. 1968. Optimization of tree-structured natural-gas transmission networks. Journal of Mathematical Analysis and Applications $\mathbf{2 4}$ 613-626.

[33] Wu, S., R.Z. Rios-Mercado, E.A. Boyd, L.R. Scott. 2000. Model relaxations for the fuel cost minimization of steady-state gas pipeline networks. Mathematical and Computer Modelling 31 197-200. 